\title{
Aero-Structural Modeling of the Truss-Braced Wing Aircraft Using Potential Method with Correction Methods for Transonic Viscous Flow and Wing-Strut Interference Aerodynamics
}

\author{
Jason Fugate* \\ Stinger Ghaffarian Technologies, Inc., Moffett Field, CA 94035 \\ Nhan Nguyen ${ }^{\dagger}$ \\ NASA Ames Research Center, Moffett Field, CA 94035 \\ Juntao Xiong \\ Stinger Ghaffarian Technologies, Inc., Moffett Field, CA 94035
}

\begin{abstract}
This paper describes an aero-structural modeling method for the Transonic Truss-Braced Wing (TTBW) aircraft using VSPAERO. A vortex-lattice model of the TTBW aircraft is developed, and a transonic and viscous flow correction method is implemented in the VSPAERO models to account for transonic and viscous flow effects. A correction method for the wing-strut interference aerodynamics is developed and applied to the VSPAERO solver. Also, a structural dynamic finite-element model of the TTBW aircraft is developed. This finite-element model includes the geometric nonlinear effect due to the tension in the struts which cause a deflectiondependent nonlinear stiffness. The VSPAERO models are coupled to the finite-element model to provide a rapid capability for aero-structural modeling and flutter analysis. A flight-optimized jig twist model is being developed and will be applied for the purpose of generating a full flight dynamic model of the TTBW aircraft.
\end{abstract}

\section{Introduction}

The demand for green aviation is expected to increase with the need for reduced environmental impact of air travel. Most large transports today operate within the best cruise L/D range of 18-20 using the conventional tube-and-wing design. This configuration has led to incremental improvements in aerodynamic efficiency over this past century. In recent years, the use of lightweight materials, such as composites, has been shown to significantly reduce structural weight and trim drag, leading to improved energy efficiency. The Boeing 787 transport is an example of a modern airframe design that employs lightweight structures. High aspect ratio wing design can provide another opportunity for further improvements in energy efficiency.

Research and development of high aspect ratio wing transport designs has placed a greater emphasis on the studies of aeroelasticity and flutter owing to the increase in the wing flexibility as the wing aspect ratio increases. These studies have sought to develop methods and tools for aeroelasticity by laying the foundation for more modern high aspect ratio wing aircraft such as the Transonic Truss-Braced Wing (TTBW). ${ }^{1,2,3}$ The concept of using truss structures to alleviate the wing root bending moment of an ultra-high aspect ratio wing has a long history in aviation with more than a decade of work focused on improving the understanding of the aeroelastic properties and structural weight penalties associated with the increase in the wing flexibility.

The Subsonic Ultra Green Aircraft Research (SUGAR) Transonic Truss-Braced Wing (TTBW) aircraft concept is a Boeing-developed N+3 aircraft configuration funded by NASA ARMD Advanced Air Transport Technologies (AATT) project. ${ }^{4,5}$ The TTBW aircraft concept is designed to be aerodynamically efficient by employing a wing aspect ratio of about 19.55, which is significantly greater than those of cantilever wing transport configurations. For example, the latest Boeing $777-\mathrm{X}$ is reported to have a wing aspect ratio of 11 . Without structural bracing, the increase in the wing root bending moment would require a significant structural reinforcement which would lead to an increase in the structural weight that would offset the aerodynamic benefit of the high aspect ratio wing. Thus, the design of a truss-braced structure is a multidisciplinary design optimization process that strives to achieve a delicate

\footnotetext{
*Aerospace Engineer, Intelligent Systems Division, jason.fugate@ nasa.gov

†Senior Research Scientist, Associate Fellow AIAA, Intelligent Systems Division, nhan.t.nguyen@ nasa.gov

$\doteqdot$ Aerospace Engineer, Intelligent Systems Division, juntao.xiong@nasa.gov
} 
balance between aerodynamic efficiency and structural efficiency. In the SUGAR configuration, the trade between the aerodynamic performance and structural design results in a truss-braced configuration with the wings braced at approximately mid-span by two main struts. In addition, two jury struts, one on each wing, provide the additional structural reinforcement. Two versions of the TTBW configurations are currently being developed by Boeing; a Mach 0.745 version and a Mach 0.8 version. Figure 1 is an illustration of the TTBW aircraft.

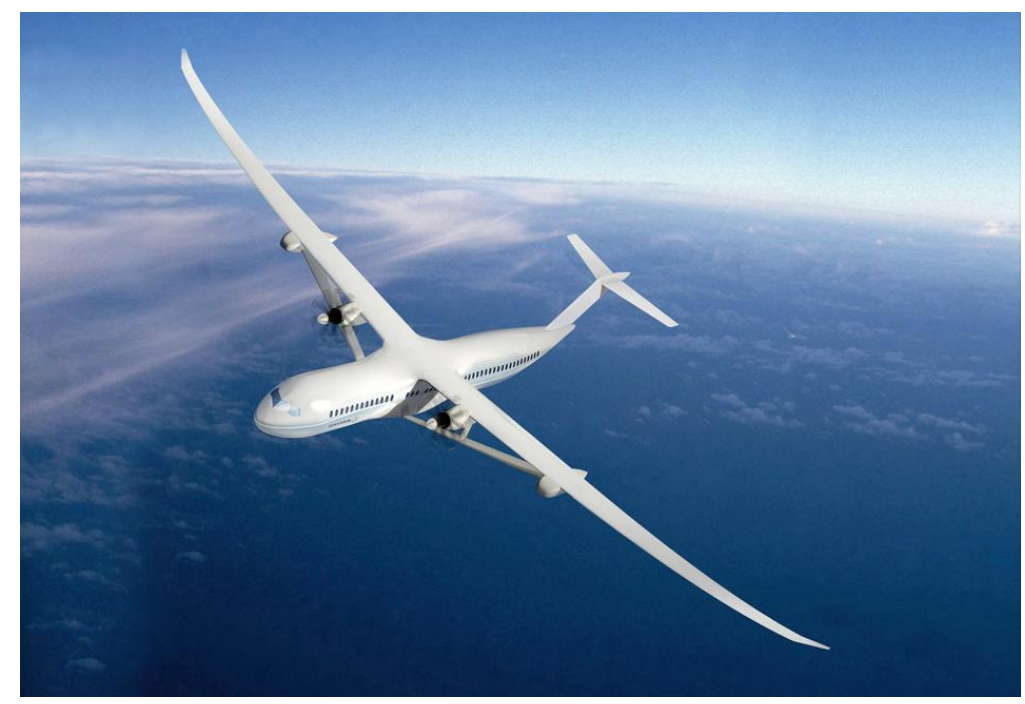

Figure 1 Boeing SUGAR Transonic Truss-Braced Wing (TTBW) Aircraft Concept

The development of the TTBW aircraft is funded by the NASA AATT project through a NRA (NASA Research Announcement) selection of Boeing Research and Technology as the lead organization. Multidisciplinary design analysis and optimization (MDAO) studies have been conducted at each stage to improve the wing aerodynamics, structural efficiency, and flight performance using advanced $\mathrm{N}+4$ turbofan engines. These MDAO studies have refined the geometry of the wing and configuration layout and have involved trade studies involving minimizing induced drag, profile drag, and wave drag due to the addition of the main strut and jury struts.

While there are on-going efforts in developing high-fidelity Reynolds-averaged Navier-Stokes (RANS) computational fluid dynamics (CFD) models of the TTBW for aerodynamic design and performance analysis, these high-fidelity CFD models are typically not coupled to the structure of the TTBW. There is a need to conduct aeroelastic analysis of the TTBW configuration to address flutter requirements and aero-structural coupling which can affect aerodynamics and vehicle stability and control. Thus, structural coupling is an important element of the TTBW development. To enable this analysis capability, one approach is to use a lower-order aerodynamic tool that can still sufficiently capture the transonic and viscous flow effects on the vehicle for coupling to a finite-element model of the TTBW structure. This approach can provide a rapid analysis capability that facilitates vehicle optimization with aeroelastic constraints such as flutter. This is a practical approach considering that currently, a vehicle optimization with flutter constraints cannot be handled by high-fidelity CFD tools due to the extremely high computational cost of running unsteady CFD simulations coupled with finite-element models. The industry standard for flutter analysis is the doublet lattice method available in NASTRAN, but this tool does not account for the transonic and viscous flow effects. Corrections to the sectional lift curve slope can be made in NASTRAN by using steady state RANS CFD solutions. During a design optimization, as the vehicle configuration changes, new steady state RANS CFD models have to be created. Thus, even this approach can still present a large computational cost.

The TTBW configuration provides a structural solution to high aspect ratio wing aircraft designs. The long slender wing employs structural bracing via the use of axially loaded strut members to provide intermediate span supports in addition to the wing root attachment. These struts generally support a portion of the span load carried by the wing and are generally loaded in tension. Under a negative-g flight condition such as during a dive, a load reversal could occur that could put the struts in compression. The compressive loading would require a design consideration for buckling 
strength. Under aerodynamic loading, an axially loaded member also experiences the normal bending and torsion generated by aerodynamic lift force and pitching moment. Aeroelasticity of an axially loaded structure undergoing transverse bending can be significantly different from that with transverse bending alone. The presence of axial loading causes the bending and torsional stiffnesses to change. A tensile loading will result in an increase in bending and torsional stiffnesses. This tension-induced stiffening effect is a geometric nonlinearity which should be captured in aeroelastic analyses of the TTBW configuration. ${ }^{11}$ Thus, the TTBW structure is inherently a nonlinear structure. A recent study has investigated the effect of axial loading of the TTBW configuration on the flutter speed. ${ }^{11}$ The study shows that the flutter speed has a nonlinear characteristic and is sensitive to the load factor or the angle of attack.

This paper presents a flutter analysis approach for the TTBW aircraft utilizing the potential flow solver called VSPAERO coupled to an in-house nonlinear finite-element code titled BEAM3D. The VSPAERO models of the TTBW include both a low-fidelity vortex-lattice model and a mid-fidelity panel model for steady-state aerodynamics. Transonic and viscous flow corrections for the steady-state aerodynamics are implemented on the vortex-lattice model using a transonic small disturbance (TSD) code called TSFOIL coupled to an in-house integral boundary layer (IBL) code. In the region near the strut attachment to the wing, the flow involves a considerable degree of interactions between the wing and the strut. A high-fidelity CFD model of the TTBW is developed using FUN3D to investigate the wing-strut interference aerodynamics for the purpose of developing a wing-strut interference aerodynamic correction method applied to the VSPAERO model. Upon applying all the necessary corrections, the VSPAERO model shows an excellent agreement with wind tunnel test data for the Mach 0.745 version of the TTBW. The BEAM3D finite-element model of the TTBW is based on a three dimensional beam theory which includes all six degrees of freedom at a node. The BEAM3D model is coupled to the VSPAERO for static aeroelastic analysis. The mass and stiffness properties of the TTBW are provided by Boeing. The BEAM3D model has been validated against NASTRAN static deflection analysis and frequency analysis. Within BEAM3D, unsteady aerodynamic mass, damping, and stiffness matrices are implemented using the Theodorsen's theory for unsteady aerodynamics and the steady-state lift curve slope, $c_{l_{\alpha}}$, for steady-state aerodynamics computed by VSPAERO. An unsteady transonic correction method to the Theodorsen's theory is proposed in this paper to better capture the changes in the amplitude and phase shift in transonic flow. This modeling approach can provide a rapid aeroelastic analysis capability for flutter analysis without incurring the high computational overhead associated with a RANS CFD-based approach.

\section{Aerodynamic Model of the Truss-Braced Wing}

\section{A. VSPAERO Model}

In order to develop a rapid aeroelastic capability for flutter analysis that facilitates a vehicle MDAO process, a lower-fidelity aerodynamic model of the TTBW is necessary. A previous effort in modeling the TTBW using the vortex-lattice code VORLAX based on the aerodynamic superposition principle has shown that low-order aerodynamic tools can provide a reasonably accurate prediction of aerodynamic performance of the TTBW. ${ }^{10}$ Due to the limitations of VORLAX in the previous effort, an improved vortex-lattice model as well as a panel model of the TTBW has been developed in VSPAERO. VSPAERO is a solver that includes both the vortex lattice method and the full panel method based on generalized vortex loops. The core VSPAERO solver is based on an agglomerated multi-pole approach, coupled with a preconditioned linear solver, to reduce solution times. Adaptive wakes, time-accurate, unsteady analyses, and propeller modeling are all supported. The latest version of VSPAERO supports a loosely coupled integration of BEAM3D finite-element code to perform steady state, aeroelastic analyses. VSPAERO is part of the OpenVSP design package and is freely available under the NASA open source license.

Two Mach 0.745 TTBW OML (Outer Mold Line) geometries are available: cruise (1g shape geometry), and jig shape geometry. The geometries for the Mach 0.745 TTBW are based on the CAD models of the 765-095-200_RJ version. The jig shape geometry is not a flight jig shape that accounts for the wing aeroelastic wash-out twist for the full-scale vehicle at the design flight condition, but is a wind tunnel model jig shape. Wind tunnel tests have been conducted in NASA Ames 11-Ft Transonic Wind Tunnel. Both geometries are modeled in VSPAERO. Figure 2 illustrates one of the TTBW geometries used in the VSPAERO models. The initial mesh for the vortex-lattice model of the TTBW in VSPAERO is shown in Figure 3 The surface triangulation mesh of the panel model is shown in Figure 4 


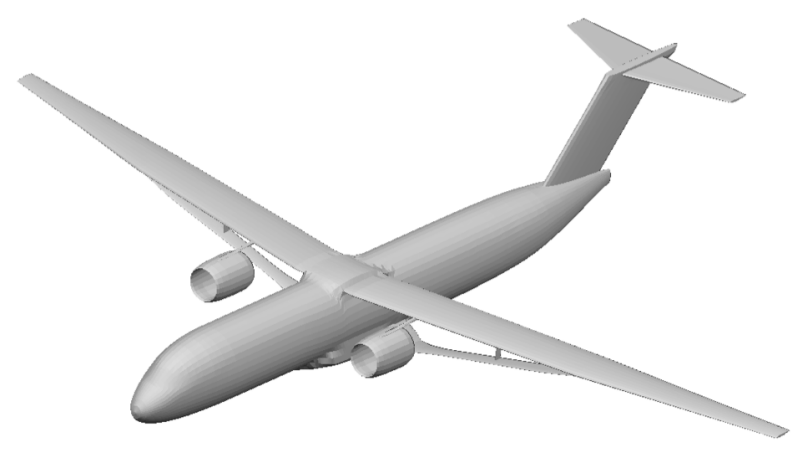

Figure 2 VSPAERO Model of TTBW

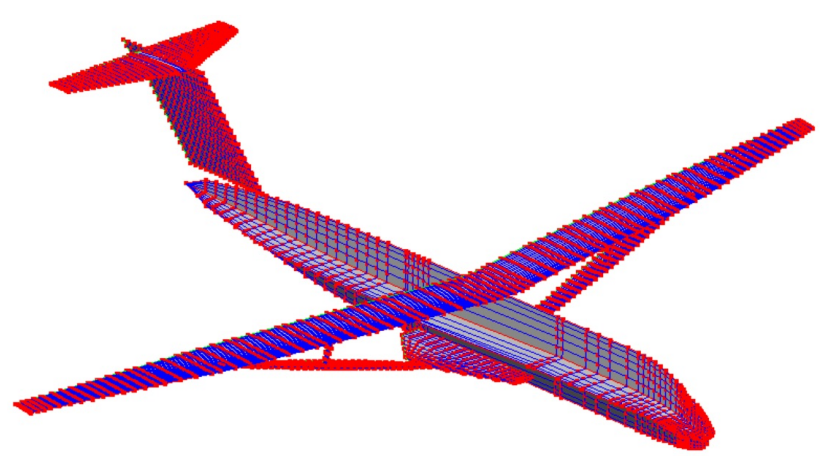

Figure 3 Initial Mesh of VSPAERO Vortex-Lattice Model of TTBW

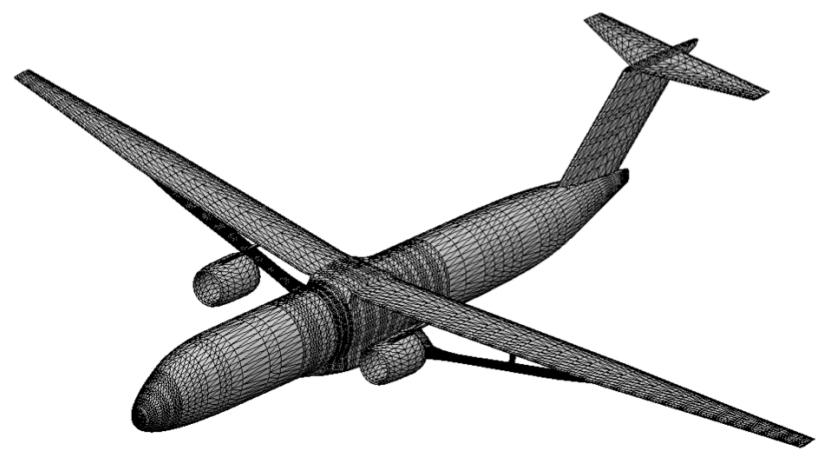

Figure 4 Surface Triangulation of VSPAERO Panel Model of TTBW

Figure 5 shows the differential pressure coefficient contour at Mach 0.7 and an angle of attack of $4^{\circ}$ for the VSPAERO vortex-lattice model. The shed wakes are shown in Figure 5 to illustrate the time-stepping wake capability in VSPAERO. Figure 6 shows the surface pressure coefficient contour computed by the VSPAERO panel model. 


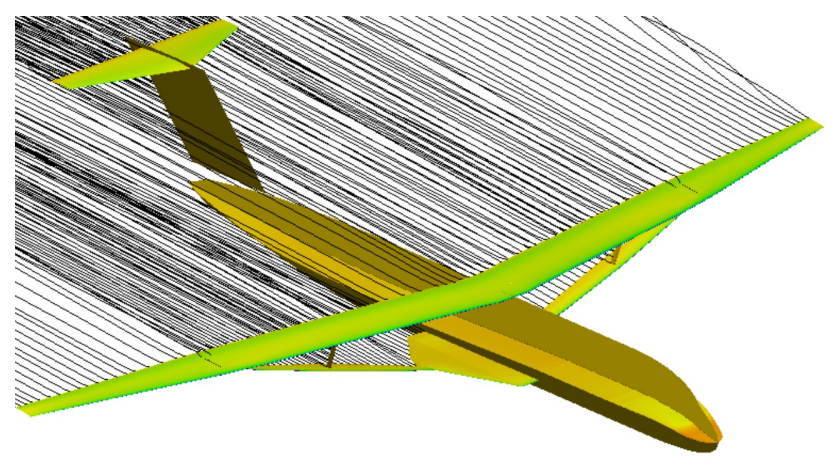

Figure 5 Differential Pressure Coefficient Contour of VSPAERO Vortex-Lattice Model of TTBW

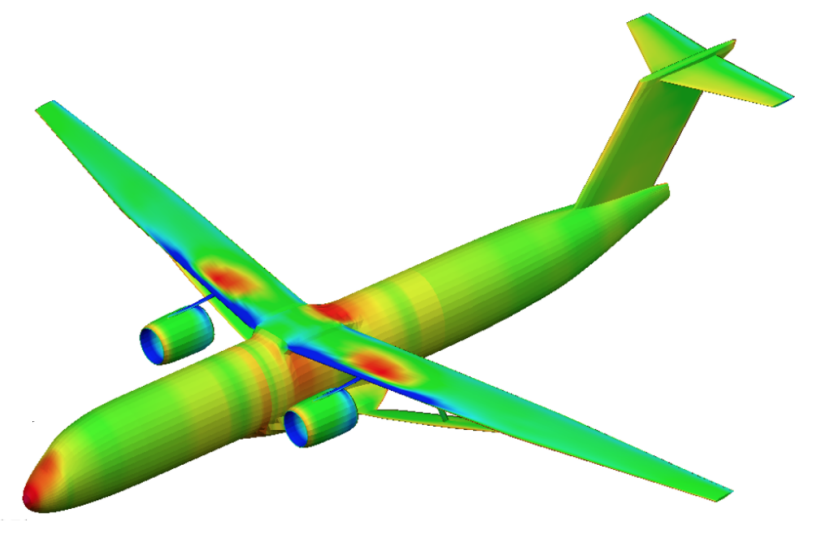

Figure 6 Surface Pressure Coefficient Contour of VSPAERO Panel Model of TTBW

The final VLM model discretization is formulated based on an intricate grid convergence study. Each aircraft component is individually analyzed. Components deemed as lifting surfaces by the interpretation standards set within the VLM software package are separated into a number of airfoils comprised of many individual nodes. The airfoils are used to geometrically determine a mean camber line, which the VLM utilizes to analyze the aerodynamic parameters of interest based on prescribed flow conditions. The number of airfoils for each component is specified based on the geometric features of that component. For instance, for the TTBW wing, it is important for cross sections to be located where the sweep changes and where other components, like the pylon, strut, and jury strut join the wing. Juncture regions are most likely to impact the mean camber line, and therefore, the aerodynamic performance of a given component. Beyond these requirements, a maximum relative distance between span stations is established for each component to ensure that all of the geometric intricacies of each component are captured. Ultimately, the combination of these strategies leads to the span station discretization scheme illustrated by Figure 7 , which is comprised of 31 individual airfoils separated by a maximum relative spacing of $\Delta \eta=0.0381$. The strut, meanwhile, is comprised of 44 cross sections to capture the complex variable taper near the root. The horizontal tail is modeled using 13 cross sections, the vertical tail requires 18 due to variable taper near the tip, the jury strut and pylon are comprised of 5 cross sections each, the nacelle uses 24 cross sections, and the fuselage uses 41 cross sections. 


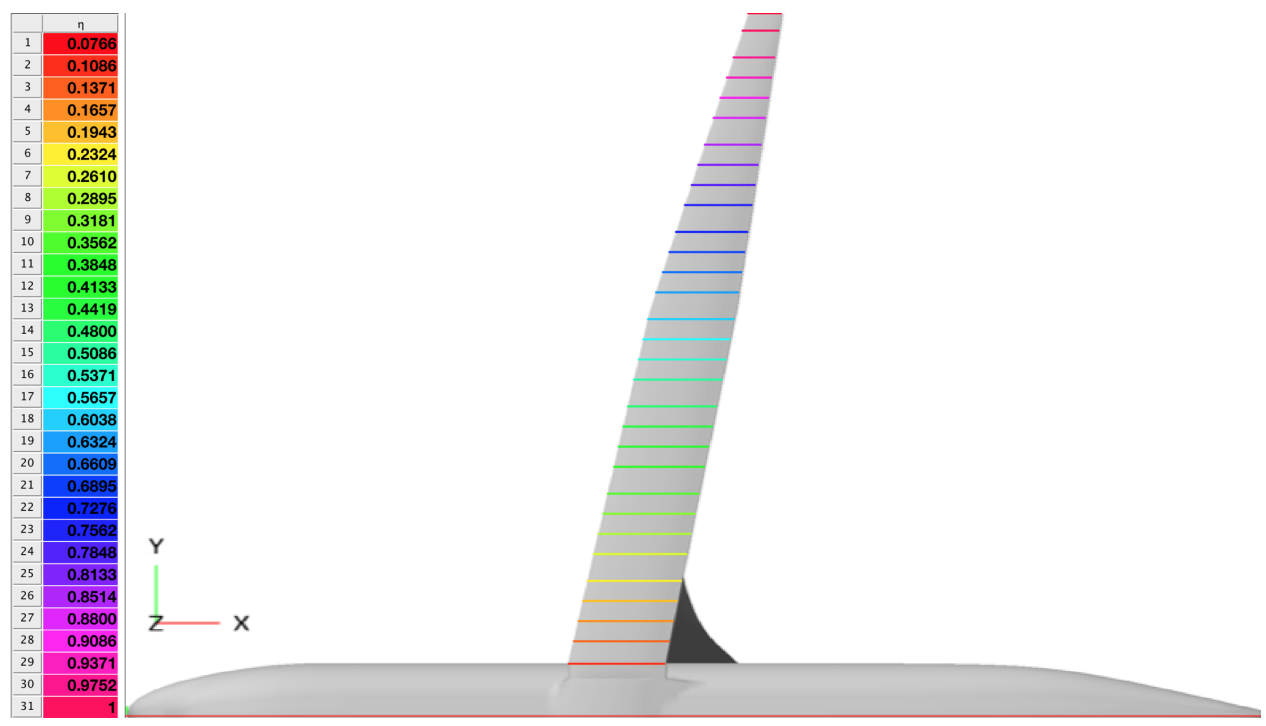

Figure 7 VSPAERO Wing Discretization Scheme

For the main lifting surfaces of the TTBW, namely the wing, horizontal tail, and strut, the number of nodes for each airfoil section is determined by increasing the number incrementally until the component lift coefficient converges. Figure 8 includes the grid convergence results for the three main lifting components of the TTBW. The ratio between the sectional lift coefficient, $c_{L}$, and the mean sectional lift coefficient for the component, $c_{L m e a n}$, is plotted as the grid is refined. Convergence is achieved when there is an increase of less that $1 \%$ between grid levels. Based on the results of this grid convergence study, 151 points per cross section are used for the wing and strut components, whereas only 75 points are necessary for the horizontal tail.

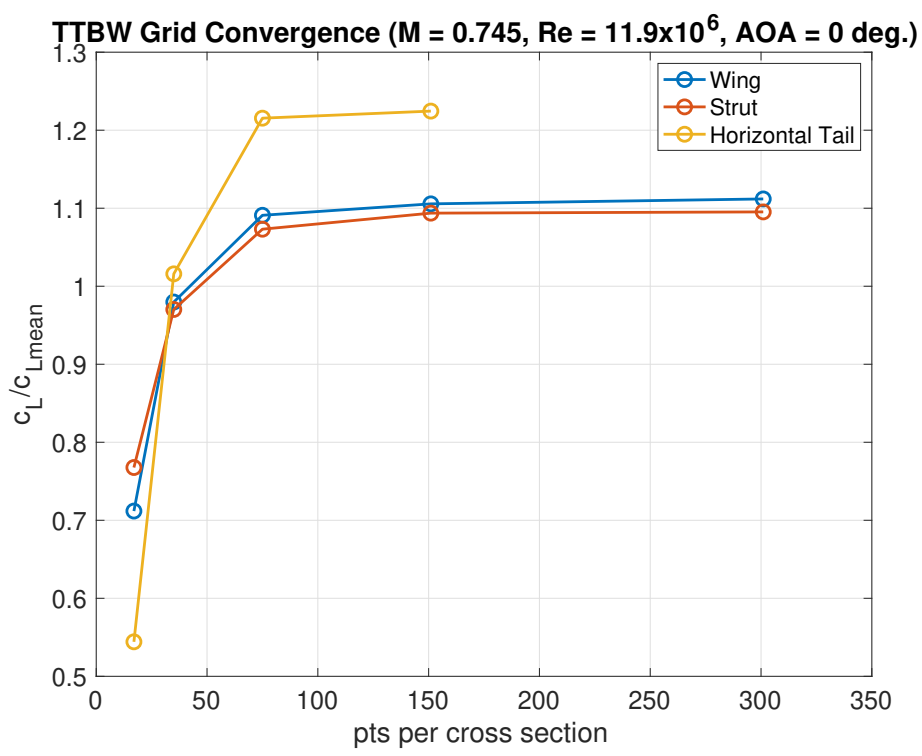

Figure 8 TTBW Grid Convergence

Minor lift-generating surfaces, such as the jury strut, vertical tail, and pylon, for which the component lift is only marginally impacted by the number of nodes per cross section, are similarly discretized into a number of airfoil sections based on a minimum separation distance. The number of nodes per cross section, however, is set to a baseline minimum of 45 nodes in order to fully capture the curvature of the mean camber line without sacrificing additional computation time by adding tedious detail. The nacelle is generated by extracting a single airfoil from the CAD model 
and rotating the airfoil 360 degrees under an approximate circular symmetry assumption. The number of sections and nodes can then be specified in a similar fashion to other minor lift-generating components. The fuselage, or lone body component, as deemed by the VLM software standards, is treated as a minor lift-generating component for the purposes of discretization.

\section{B. Transonic and Viscous Flow Correction}

Because of the missing transonic effect in the linear potential flow method, a method for transonic and viscous corrections has recently been developed. ${ }^{13,14,15}$ In this method, a full-configuration aerodynamic model can be based on the vortex-lattice method. The wing is discretized into several spanwise sections at which the section lift coefficients computed by the potential flow method are used to correct for the transonic and viscous flow effects. ${ }^{14,15}$ The transonic and viscous correction method is an iterative process to compute the incremental section lift coefficient due to transonic and viscous flow by a virtual re-twist of the individual wing sections to account for the accompanied change in the effective local angle of attack to produce the incremental section lift coefficient. ${ }^{15}$ Implementation of the correction begins by initializing the virtual twist angle due to transonic and viscous corrections, $\gamma(y)$, to zero. The effective 2D angle of attack is then calculated for each airfoil using:

$$
\alpha_{2 D}(y)=\alpha_{0}(y)+\frac{c_{l_{V L M}}(y)}{c_{l \alpha}}-\gamma(y)
$$

where $\alpha_{2 D}$ is the effective airfoil angle of attack, $\alpha_{0}$ is the the airfoil zero-lift angle of attack, $c_{l_{V L M}}$ is the sectional lift coefficient obtained via VSPAERO, and $c_{l \alpha}$ is the 2D lift curve slope corrected for sweep as follows:

$$
c_{l \alpha}=\frac{2 \pi}{\sqrt{1-M_{\Lambda}^{2}}} .
$$

Here, $M_{\Lambda}$ is the Mach number based on the mid-chord sweep angle. Each airfoil is analyzed by the TSD/IBL model at the effective angle of attack. The transonic flow correction is handled by a transonic small disturbance (TSD) code called TSFOIL. ${ }^{16}$ This code is loosely coupled to an in-house integral boundary layer (IBL) code to correct for the viscous flow interaction with the transonic shock on an airfoil. ${ }^{13}$ Optionally, the correction method is performed using the 2D Euler CFD code MSES with an integral boundary layer method developed by Mark Drela. ${ }^{17}$ The virtual twist angle is then updated for each section according to:

$$
\gamma_{i+1}(y)=\gamma_{i}(y)+\frac{c_{l_{2 D_{i}}}(y)-c_{l_{V L M_{i}}}(y)}{c_{l \alpha}},
$$

where $c_{l_{2 D_{i}}}$ corresponds to the sectional lift coefficient calculated by TSFOIL for iteration $i$.

The coupling and iterative update process is repeated until the VLM section lift and the 2D lift from the TSD/IBL model converge for all sections. Wave and friction drag are calculated by the TSD/IBL model, whereas lift, pitching moment, and induced drag are calculated by the VLM model. The flow chart of the transonic and viscous flow correction method is shown in Figure $9{ }^{15}$ An extensive validation of the transonic and viscous flow correction method has been performed to compare the method against the OVERFLOW results for a 2D airfoil, and when used with a vortexlattice model against LAVA, a RANS CFD code developed at NASA Ames, and the Euler CFD code CART3D without the viscous flow correction for a full configuration aircraft. The agreement in the $L / D$ result for the NASA Generic Transport Model (GTM) ${ }^{18}$ is less than $4 \%$ of the LAVA-computed value, as shown in Figure 10.

Additionally, the flow correction method is validated by comparing TSFOIL calculated sectional $c_{p}$ data for the TTBW at multiple span stations to results obtained using FUN3D. These comparisons can be seen in Figure 11. Note that at the inboard and outboard span stations, the two methods compare quite well; however, near the wing-strut juncture, TSFOIL fails to capture the suction peak that is induced on the lower surface due to wing-strut aerodynamic interference. The major advantage of using vortex-lattice method coupled to transonic and viscous corrections is the 
computational efficiency of the method, which is several orders of magnitude faster than RANS CFD. This computational efficiency becomes highly important when the potential flow solver is coupled to a structural finite-element model for aero-structural modeling or flutter analysis, which adds an additional computational overhead to the aerodynamic modeling.

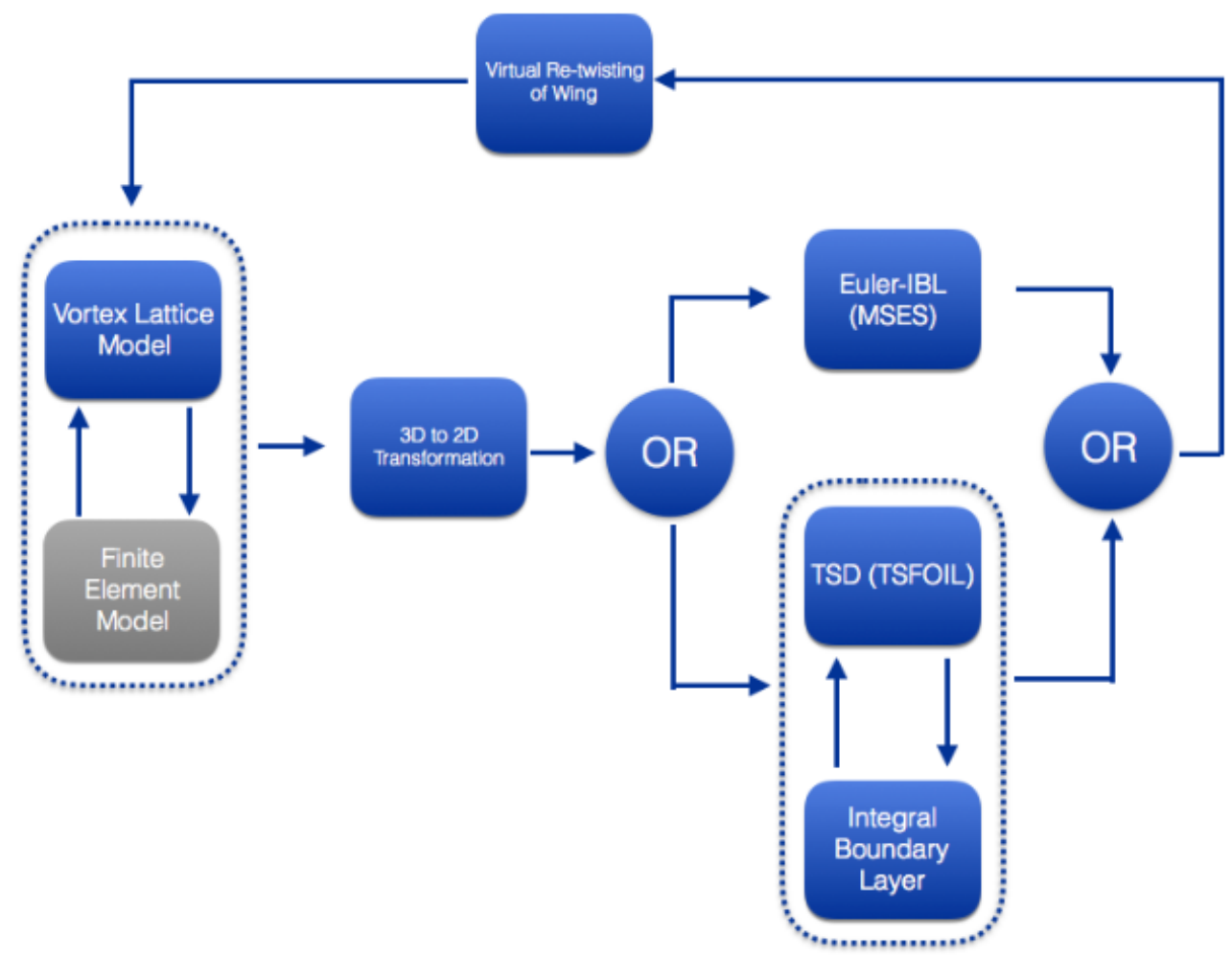

Figure 9 Transonic and Viscous Correction Flow Chart

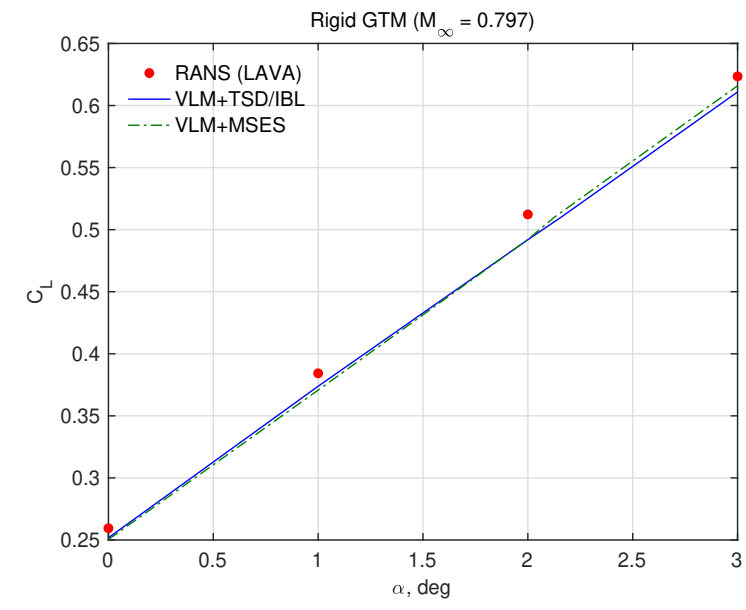

(a) Lift Curve

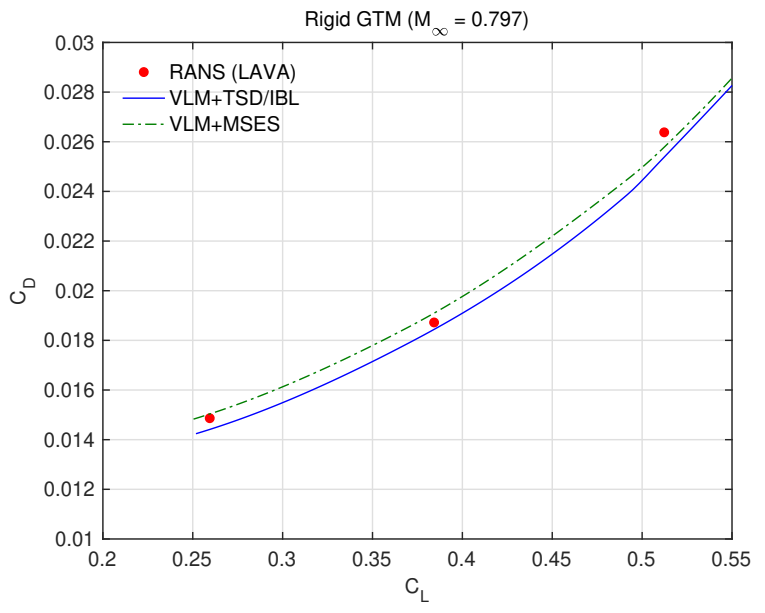

(b) Drag Polar

Figure 10 Lift Curve and Drag Polar for the Rigid Generic Transport Model Computed by TSFOIL/Integral Boundary Layer and MSES Coupled to VORLAX Compared with LAVA RANS Results 

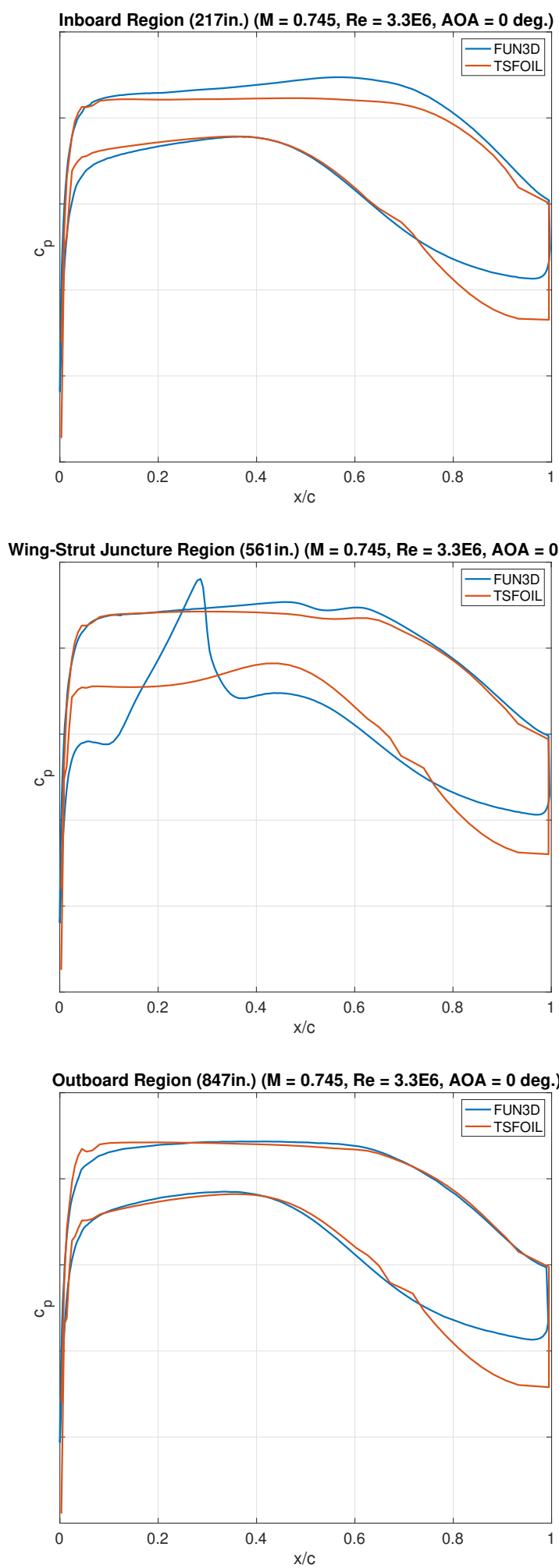

Figure 11 Sectional $c_{p}$ Distribution Comparisons between TSFOIL and FUN3D for TTBW 


\section{Wing-Strut Interference Aerodynamic Correction}

The transonic and viscous flow corrections using the TSD/IBL method are generally valid for a single-element airfoil. The TTBW configuration is a complex geometry that includes a strut juncture region where the effect of interference aerodynamics can influence the overall aerodynamic performance of the aircraft. As the strut approaches the wing from below, the transonic and viscous flow corrections using the TSD/IBL method are no longer valid due to the interactions between the wing airfoil and strut airfoil, as can be seen in the plot of pressure distribution in Figure 11. To account for these interference aerodynamics, CFD models of the wing-strut configuration and the wing-alone configuration of the jig shape TTBW geometry are developed using FUN3D, as shown in Figure 12 To isolate the interference aerodynamic effect for the wings and struts alone, the engines and the horizontal tails are removed from the models. The Roe's flux-difference splitting scheme and the Spalart Allmaras turbulence model are used in the study. Figure 13 shows the grids for the full configuration with the engine, which is used for the grid independent study and for validation. The total number of nodes is 90 million. In this study, the mesh is comprised of tetrahedral elements and prism layer near the wall. The prism layer is used to resolve the turbulent boundary layer.

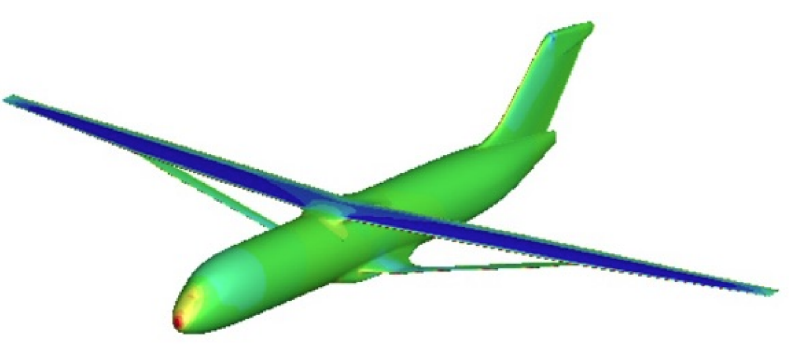

(a) Jig Shape Wing-Strut Configuration

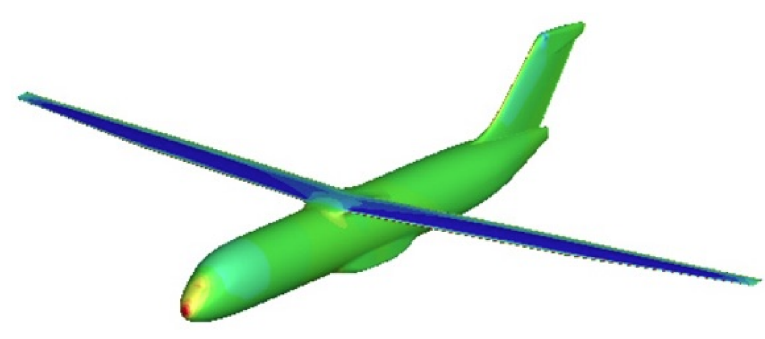

(b) Jig Shape Wing-Alone Configuration

Figure 12 TTBW Geometric Configurations for Interference Analysis

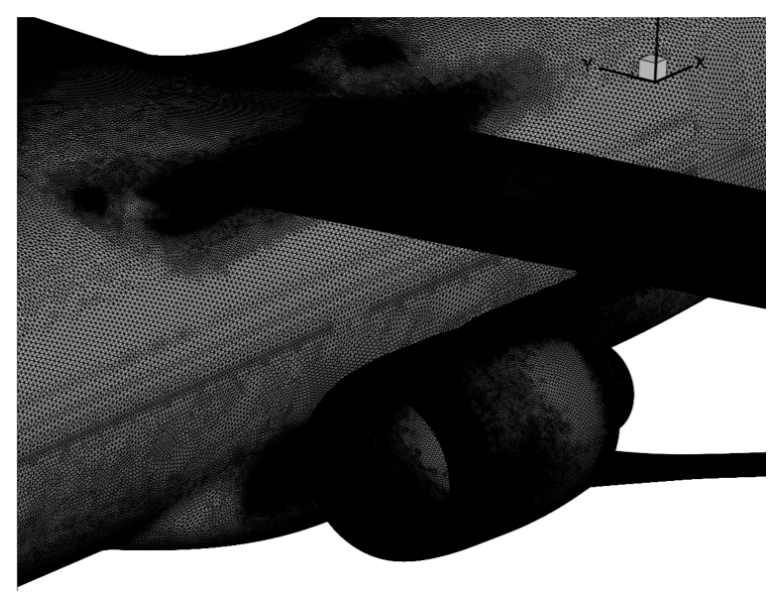

Figure 13 TTBW CFD Mesh

Surface pressure coefficients are computed for both configurations at various wing stations for Mach $0.3,0.745$, 0.8 , and 0.85 . Figure 14 shows the surface pressure coefficients at the wing station immediately inboard from the strut juncture at an angle of attack of $2^{\circ}$. By comparing the wing-strut data to the wing-alone data (not shown), it is seen that the presence of the strut induces a suction peak along the lower surface of the wing near the wing-strut juncture. The interference aerodynamic effect is seen to become increasingly significant as the Mach number increases and is most pronounced on the lower surface, where the suction peak occurs. 
A wing-strut interference correction method is developed to correct the VSPAERO model coupled to the TSD/IBL approach. The correction method is applied to the VSPAERO+TSD/IBL model to update the section lift, drag, and pitching moment coefficient of each wing section. The correction is also applied to the section lift curve slope, $c_{L \alpha}$, and the location of the aerodynamic center, $x_{a c} / c$, which are two important parameters for flutter analysis.

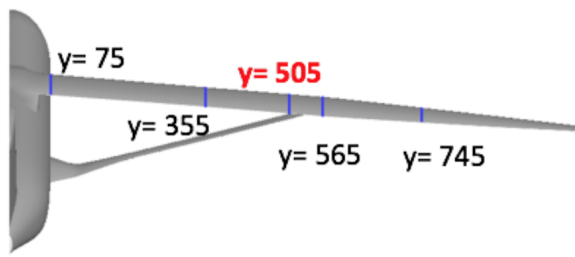

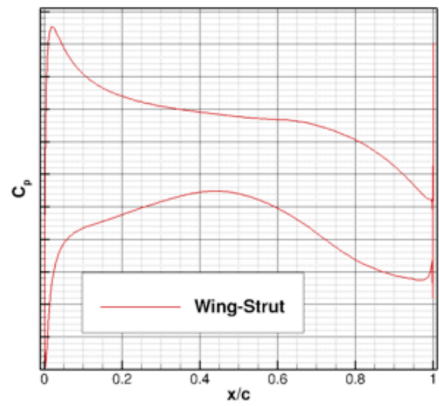

$\mathrm{Ma}=\mathbf{0 . 3 0}$

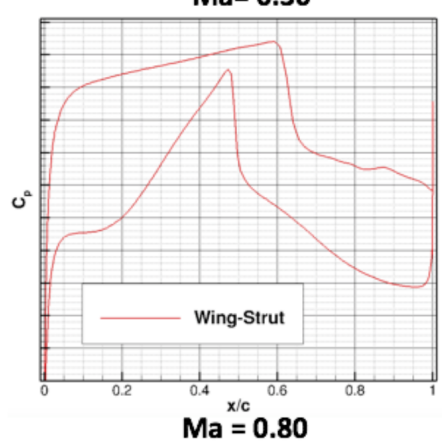

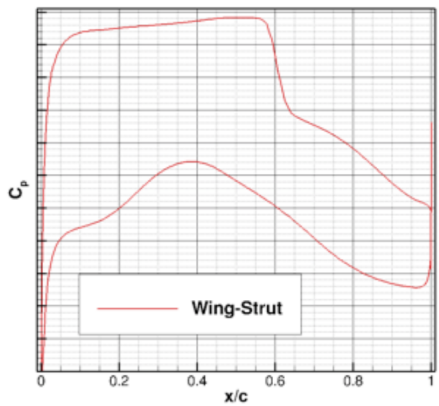

$\mathrm{Ma}=\mathbf{0 . 7 4 5}$

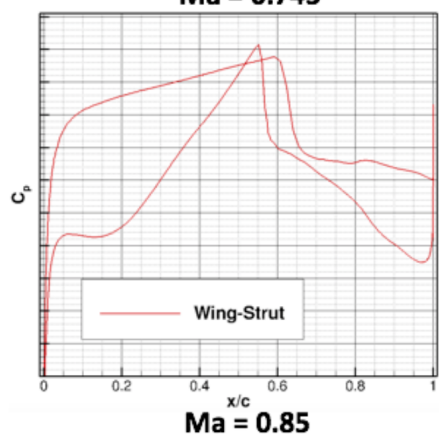

Figure 14 Pressure Coefficients at Near-Juncture Wing Station as Function of Mach Number for $\alpha=2^{\circ}$

The interference correction, $\Delta c_{I C}$, that is applied to the model is calculated using the following equation:

$$
\Delta c_{I C}=d c_{F U N 3 D}-d c_{V S P A e r o+T S D / I B L},
$$

where $c$ represents a key aerodynamic parameter such as $c_{L}, c_{D}, c_{M_{q c}}, c_{L \alpha}$, and $x_{a c}$ and $d c$ represents the change in the given parameter between the two geometric configurations.

Figure 15 shows the lift distribution and the sectional lift coefficient correction along the wing span for a Mach number $=0.745, C_{L}=0.4536$, and Reynolds number $=3.3$ million. The presence of the strut enhances the shock wave on the upper surface of the wing at inboard wing stations, which increases the lift coefficient. There is an abrupt change in the lift coefficient near the wing-strut juncture location, which is caused by the suction peak on the lower surface of the wing. The lift coefficient difference decreases toward the outboard span stations of the wing where the interference effects are diminished. For the implementation of the wing-strut interference correction, piecewise polynomials are used to fit the data for the inboard wing stations. Near the strut and the outboard wing stations, two discontinuous linear fits are used where the discontinuity occurs at the wing-strut juncture location.

Figure 16 shows the drag distribution and the sectional drag coefficient correction along the wing span for Mach number $=0.745, C_{L}=0.4536$, and Reynolds number $=3.3$ million. There is an abrupt change in the drag coefficient near the wing-strut juncture location for FUN3D simulation results, which is caused by the suction peak on the lower surface of the wing. The VSPAERO+TSD/IBL model could not capture the change of the sectional drag coefficient near the wing-strut juncture location due to its limitations. In this case, piecewise polynomials are used to fit the drag coefficient correction distribution for the inboard wing stations and near the strut, and a linear fit is used for the outboard wing stations.

Figure 17] shows the pitching moment distribution and the sectional pitching moment coefficient correction along the wing span direction for Mach number $=0.745, C_{L}=0.4536$, and Reynolds number $=3.3$ million. The pitching moment 
is calculated about the quarter chord location. The presence of the wing-strut juncture only changes the pitching moment coefficient near the juncture location because the suction peak on the lower surface of the wing reshapes the pressure distribution on the wing. A piecewise polynomial is used to fit the sectional pitching moment coefficient correction near the strut, and two linear fits are used for the inboard and outboard wing stations.

It should be noted that for the interference correction procedure, piecewise polynomials are used to fit the correction data. A scale is established for each segment so that the corresponding interference correction can be calculated at any given span station based on the aircraft lift coefficient. Certain regions along the span, which are fit by the linear curves depicted in red, are found to have no direct correlation to the lift coefficient. Thus, the interference effect in these regions is treated as constant with varying aircraft lift coefficient. It should also be noted that these curve fitting techniques allow for scalability based on aircraft lift; however, changes to the Mach number would necessitate the establishment of new scales. The impact of varying Reynolds number on aerodynamic interference is found to be marginal via FUN3D; therefore, the established relationships are deemed sufficient for all Reynolds numbers investigated.

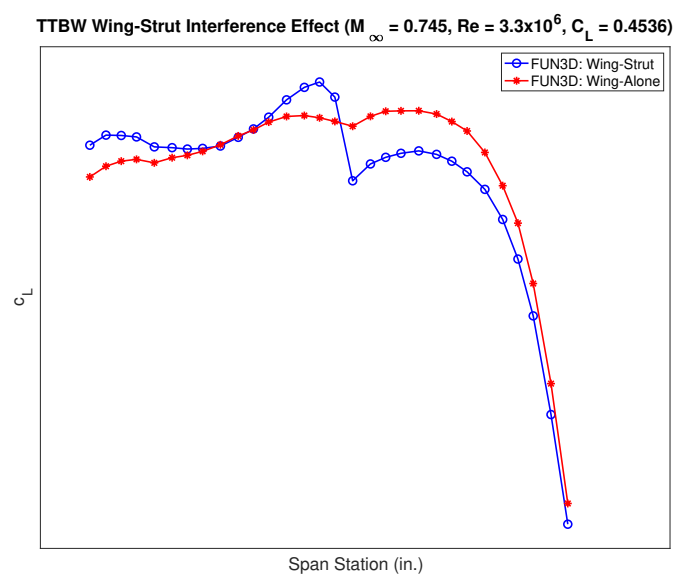

(a) Lift Coefficient Distribution - FUN3D

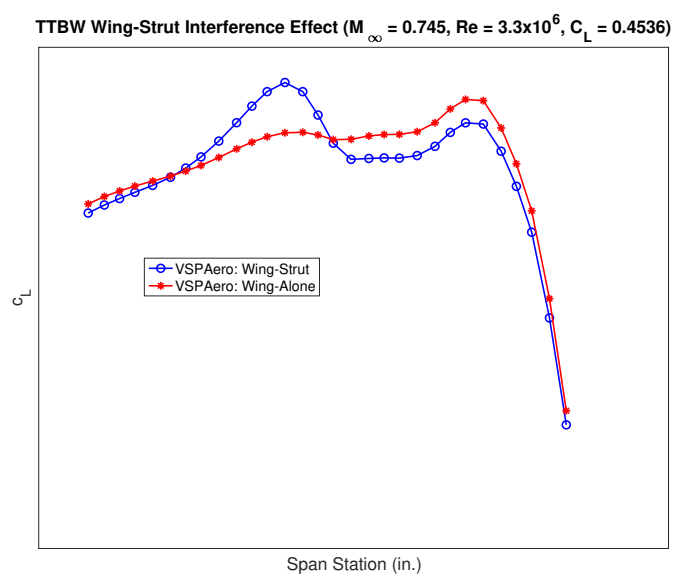

(b) Lift Coefficient Distribution - VSPAERO

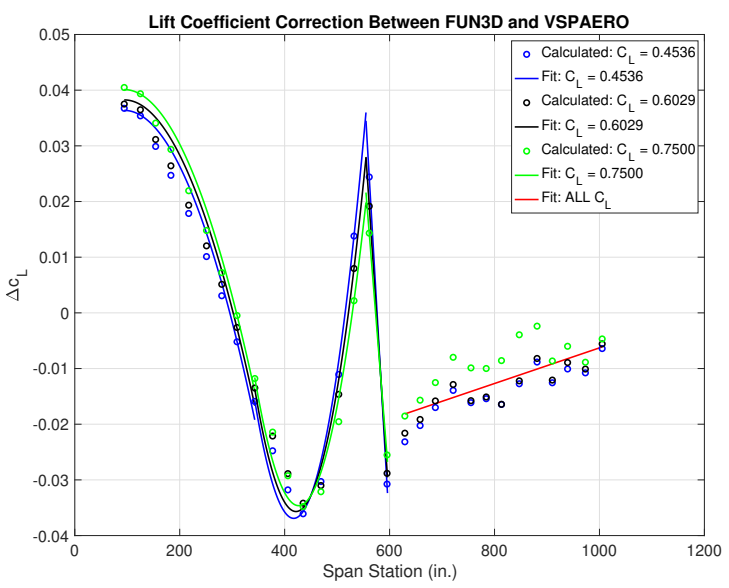

(c) Lift Coefficient Correction Distribution

Figure 15 Lift Coefficient and Lift Coefficient Correction Distribution 


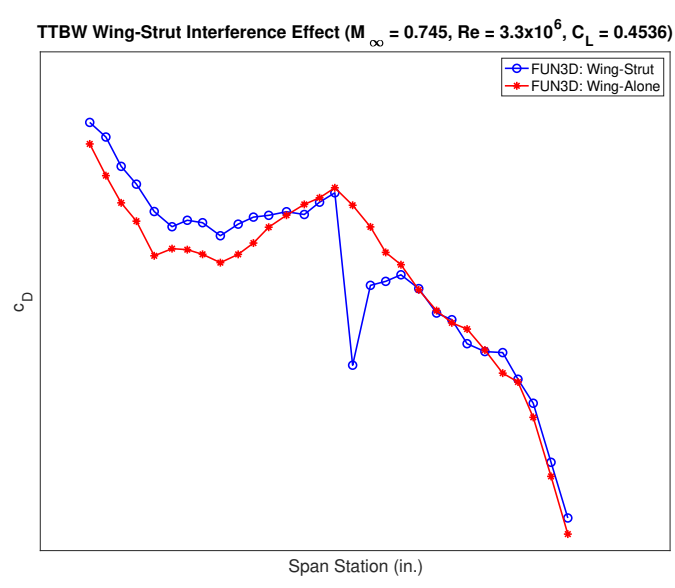

(a) Drag Coefficient Distribution - FUN3D

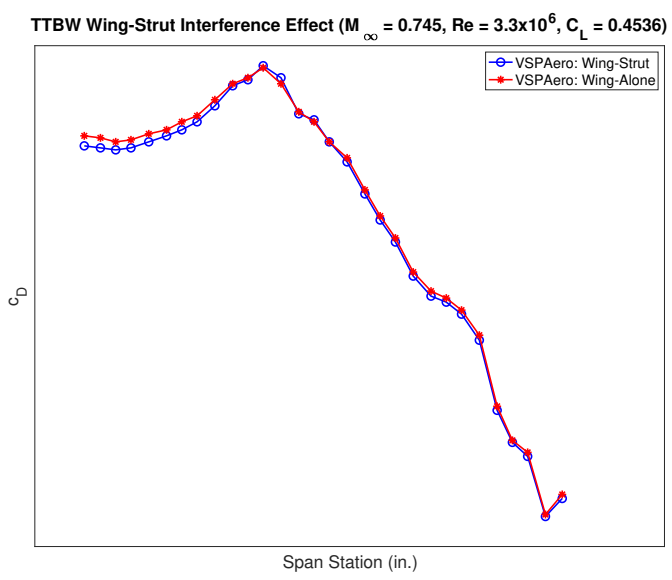

(b) Drag Coefficient Distribution - VSPAERO

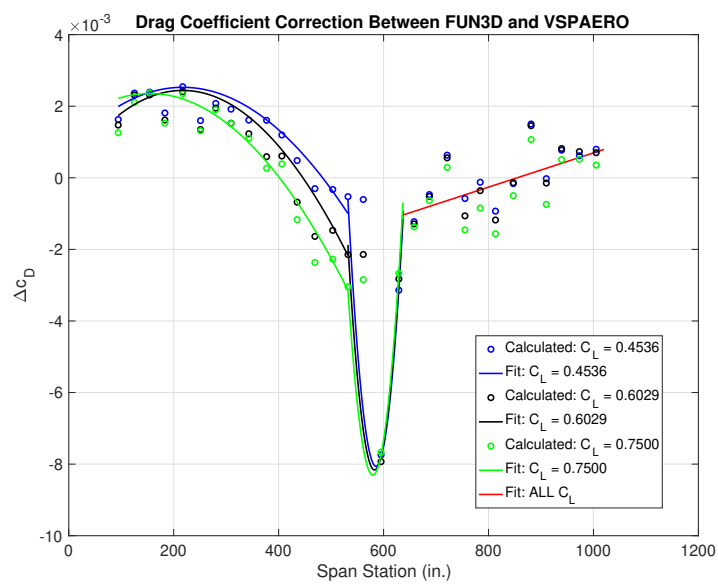

(c) Drag Coefficient Correction Distribution

Figure 16 Drag Coefficient and Drag Coefficient Correction Distribution

The interference effects on two other key aerodynamic parameters, namely the sectional lift curve slope, $c_{L \alpha}$, and sectional aerodynamic center location $x_{a c}$ are also investigated. Figure 18 shows sectional $c_{L \alpha}$ data and the corresponding correction for wing-strut interference, while Figure 19 shows the aerodynamic center data and the corresponding correction for wing-strut interference. For the case of $c_{L \alpha}$, it can be seen that a sharp drop in the correction occurs near the wing-strut juncture, while the remaining span stations on the wing experience a relatively small correction. The aerodynamic center location is found to be shifted very slightly forward near the juncture and slightly backward inboard of the juncture where the presence of the strut still has a noticeable impact on the aerodynamic center location. A numerically complex piecewise spline could be used to fit the $c_{L \alpha}$ and $x_{a c}$ interference correction data along the wing span. The applied correction for the $x_{a c}$ could be assumed as constant for the various flight conditions being simulated as long as Mach number is maintained. 


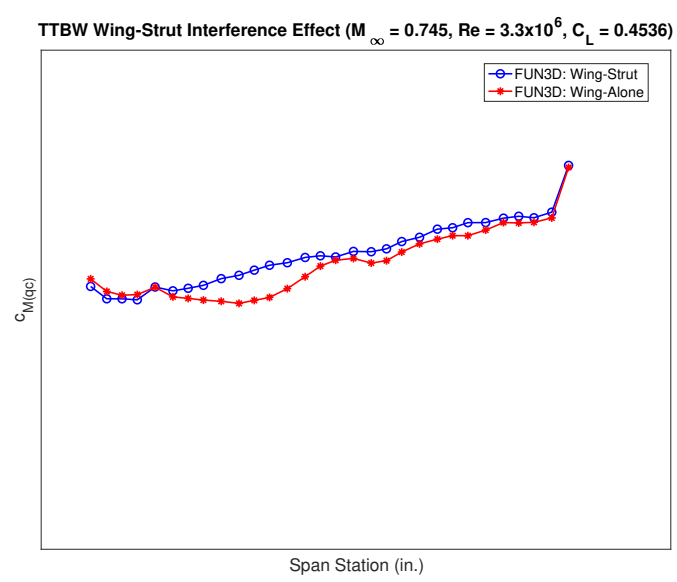

(a) Moment Coefficient Distribution - FUN3D

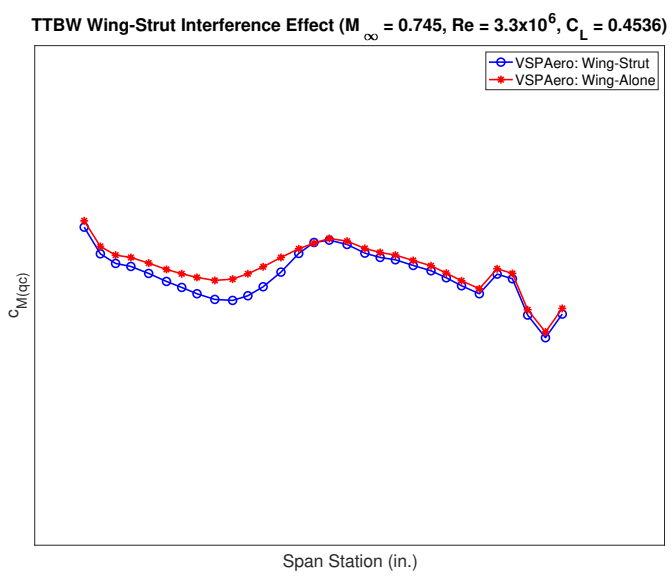

(b) Moment Coefficient Distribution - VSPAERO

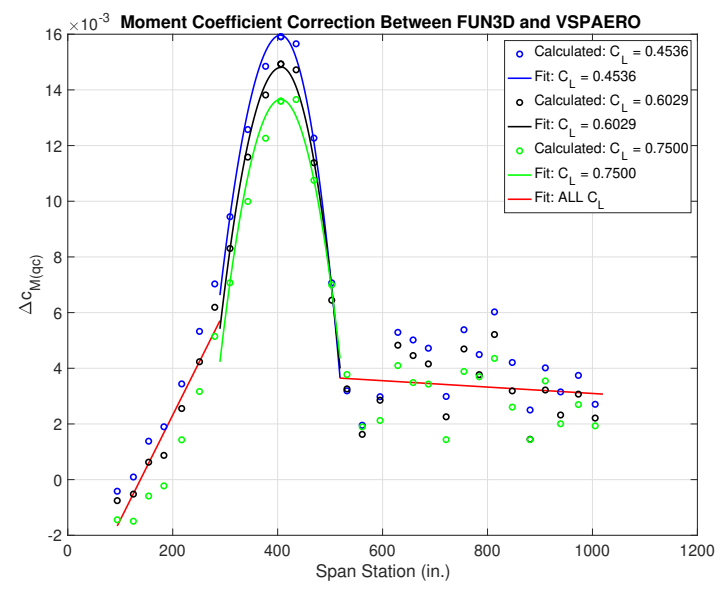

(c) Moment Coefficient Correction Distribution

Figure 17 Moment Coefficient and Moment Coefficient Correction Distribution

As can be seen, near the strut juncture region, there is a significant change of the aerodynamic performance. The wing-strut interference correction is generally small at Mach 0.745, but can become larger as the Mach number increases. With the interference correction terms applied, the VSPAERO+TSD/IBL model is used for the aerodynamic analysis of the cruise $1 \mathrm{~g}$ shape TTBW geometry and for the aero-structural analysis of the jig shape TTBW geometry. ${ }^{21}$ 


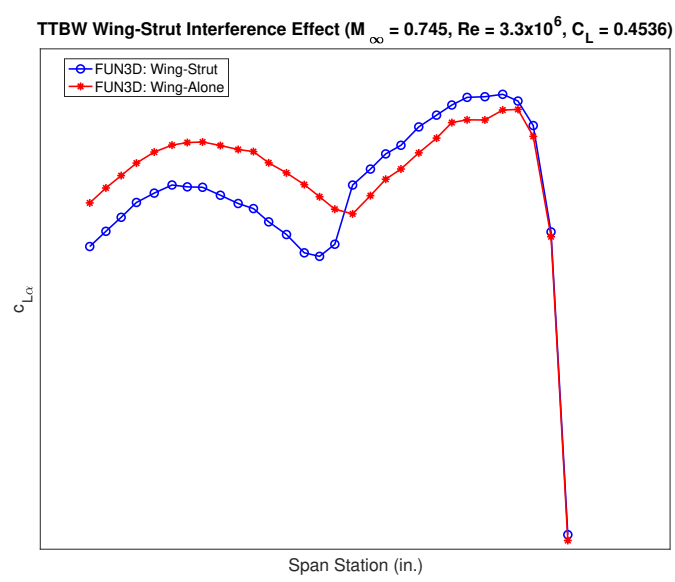

(a) Lift Curve Slope Distribution - FUN3D

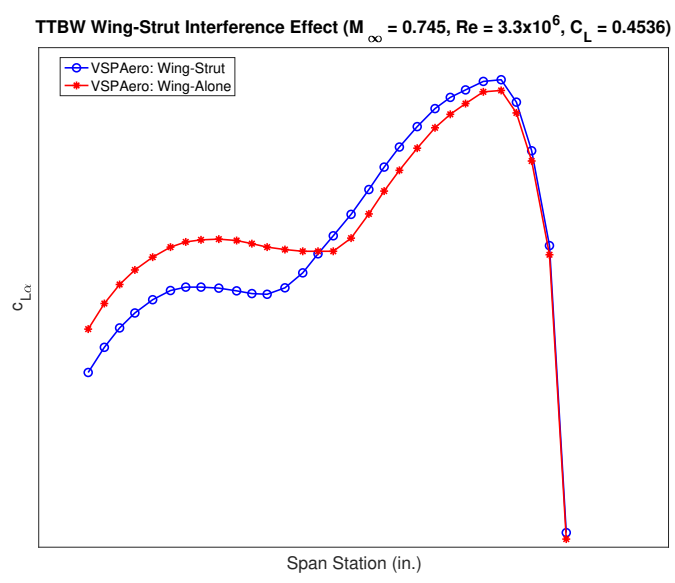

(b) Lift Curve Slope Distribution - VSPAERO

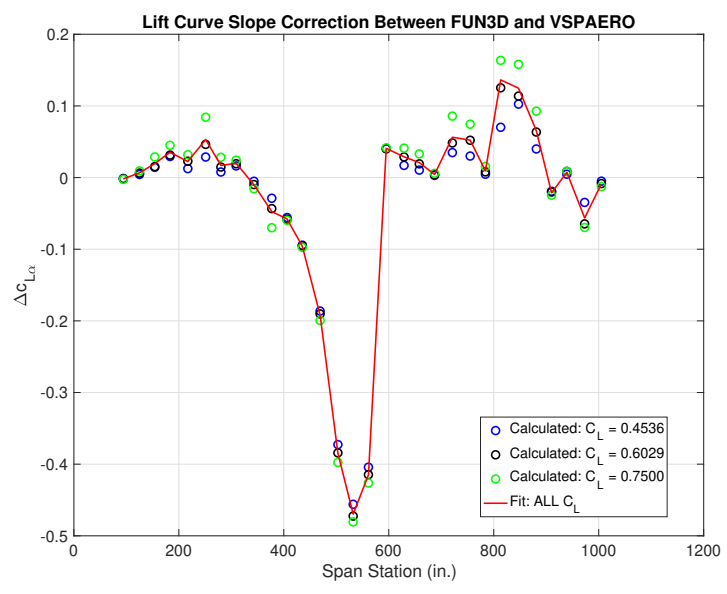

(c) Lift Curve Slope Correction Distribution

Figure 18 Lift Curve Slope and Lift Curve Slope Correction Distribution 


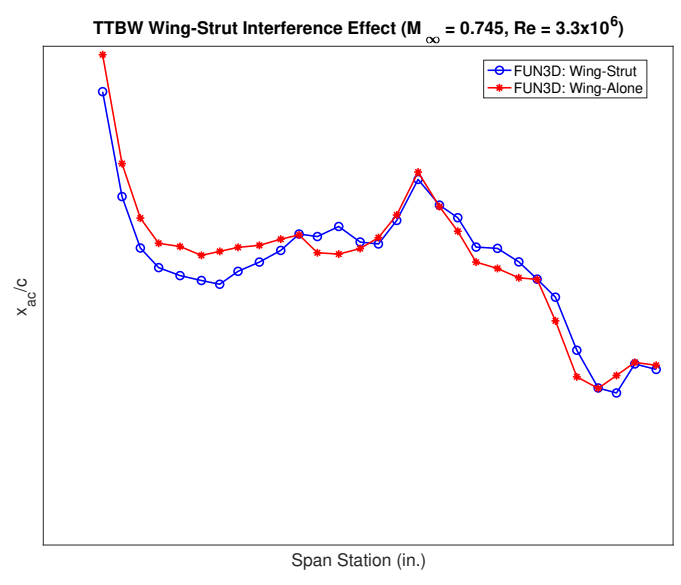

(a) Aerodynamic Center Distribution - FUN3D

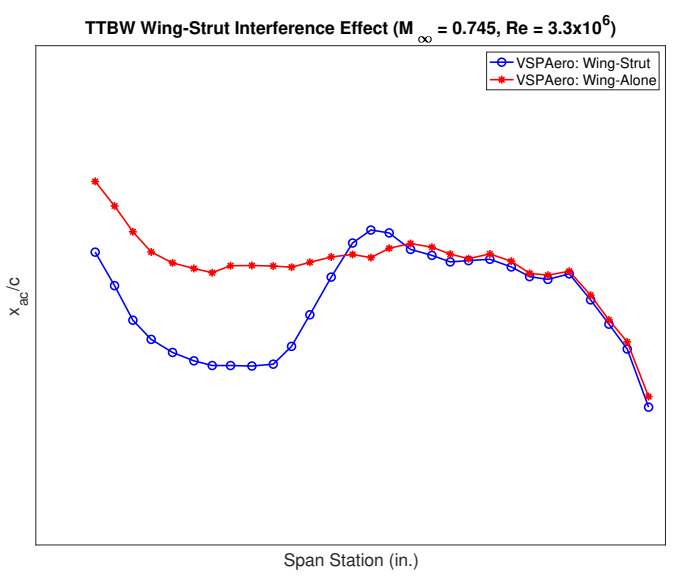

(b) Aerodynamic Center Distribution - VSPAERO

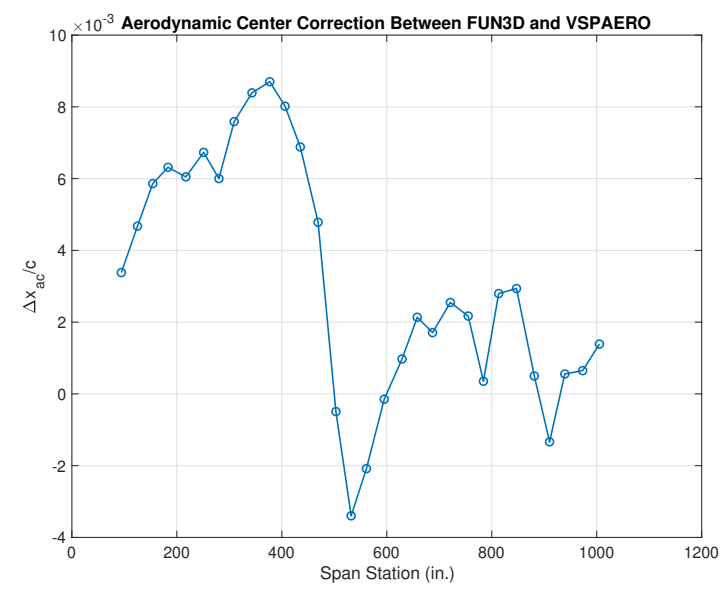

(c) Aerodynamic Center Correction Distribution

Figure 19 Aerodynamic Center and Aerodynamic Center Correction Distribution

\section{Aerodynamic Analysis of Cruise Shape Geometry}

The VSPAERO vortex-lattice model is used for the aerodynamic analysis of the cruise shape TTBW geometry. The VSPAERO panel model is not used since it has not reached the same level of maturity as the VSPAERO vortex-lattice model. Capabilities to couple the VSPAERO panel model to the BEAM3D finite element model and the TSD/IBL correction are still not yet available. In addition, a CFD model of the full- configuration cruise shape TTBW geometry is developed in FUN3D. Wind tunnel test data of the cruise shape geometry in NASA Ames 11-Ft Transonic Wind Tunnel are available for validation of the VSPAERO and FUN3D models. Test data from Run 380 at Mach 0.745 and Reynolds number of 2.6 million based on the mean aerodynamic chord (MAC) with full wind tunnel model corrections are used for validation.

Figure 20 shows the plots of the lift and drag coefficients computed by VSPAERO for Mach 0.745 and a Reynolds number of 2.6 million with and without all the corrections as well as FUN3D. These computed results are compared to Run 380 wind tunnel data. While the lift coefficient is somewhat under-predicted, the drag coefficient computed by VSPAERO alone is mostly induced drag, and therefore it is substantially under-predicted. With all the corrections applied to the VSPAERO model for transonic viscous flow and wing-strut interference aerodynamics, the lift and drag coefficients match remarkably well to the wind tunnel data, although there is a small discrepancy in the drag polar at 
lower lift coefficients. For comparison, the FUN3D results are also plotted. The lift coefficient computed by FUN3D is in less agreement with the wind tunnel data and is higher than that computed by VSPAERO with all the corrections applied. The drag polar computed by FUN3D shows an excellent agreement with the wind tunnel data.

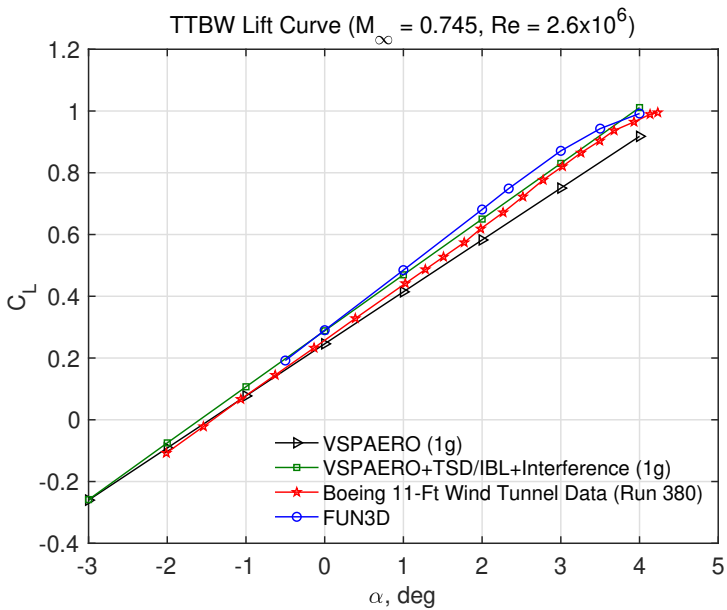

(a) Lift Curve

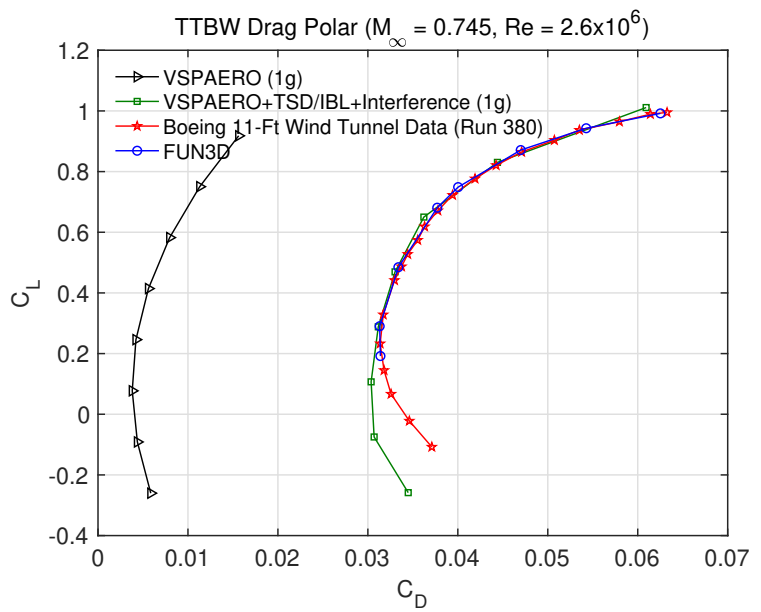

(b) Drag Polar

Figure 20 TTBW Lift Curve $C_{L}$ vs. $\alpha$ and Drag Polar $C_{L}$ vs. $C_{D}$ at Mach 0.745

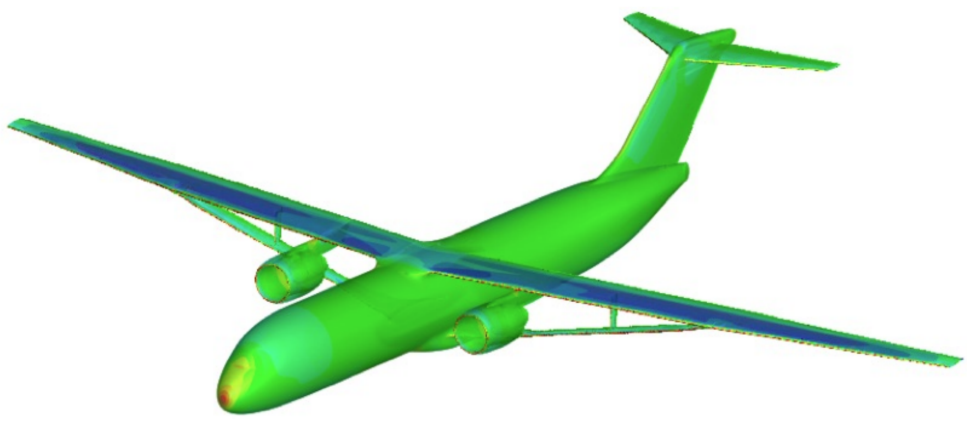

Figure 21 TTBW Pressure Coefficient Contour Computed by FUN3D at Mach 0.745 and $C_{L}=0.73$

Figure 21 is the plot of the pressure coefficient contour computed by FUN3D at Mach 0.745 and the design lift coefficient $C_{L}=0.73$. Figure 22 shows the plot of the computed $L / D$ ratios as a function of the lift coefficient from VSPAERO, FUN3D, and wind tunnel data. All the three curves of $L / D$ ratios are in very good agreement. The excellent agreement between the VSPAERO model and the wind tunnel data thus has validated the aerodynamic modeling approach. 


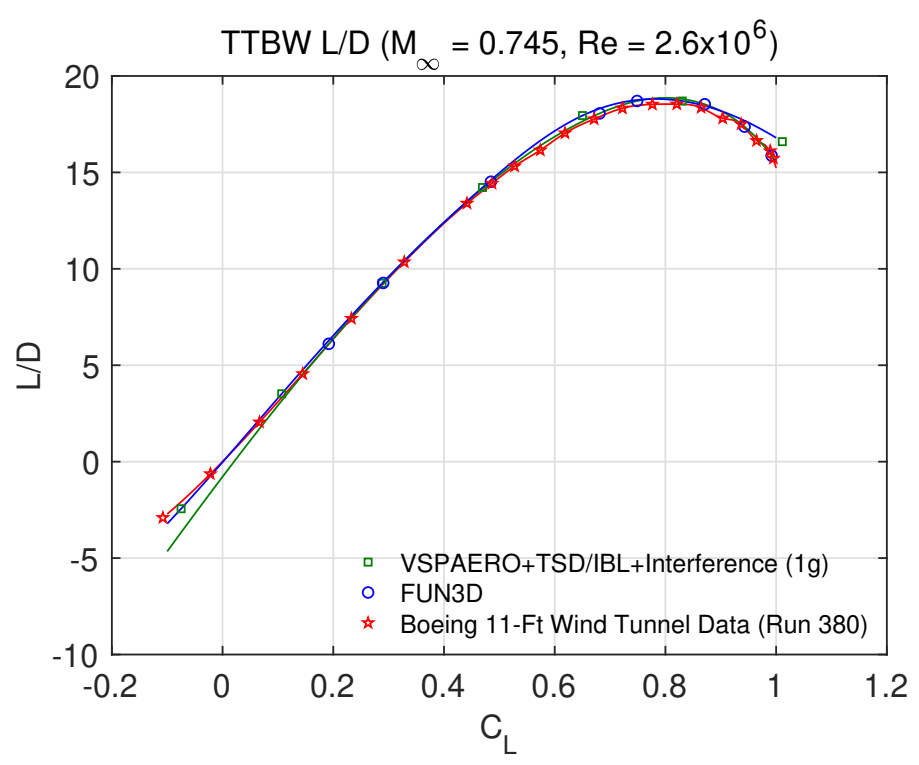

Figure 22 TTBW $L / D$ at Mach 0.745

\section{Finite-Element Model of the Truss-Braced Wing}

\section{A. BEAM3D Model}

BEAM3D is an in-house finite-element code that is used to model a general wing structure using beam, rod, and rigid elements. The code contains a number of analysis modules: a static deflection analysis module, an eigenvalue analysis module, a flutter analysis module, and an aeroservoelastic flight dynamic simulation module. The static deflection analysis module can be coupled to a flow solver such as VORLAX, VSPAERO, and CART3D to perform aero-structural and static aeroelastic deflection analyses. The eigenvalue analysis module computes the structural dynamic frequencies and the normal mode shapes of a wing structure. The flutter analysis module computes the frequencies and damping of the wing aeroelastic modes and performs a flutter speed search in the frequency domain using the Theodorsen's theory for unsteady aerodynamics or in the time domain using the $\mathrm{R}$. T. Jones approximation to generate the aerodynamic mass, damping, and stiffness. The aeroservoelastic flight dynamic simulation module provides the capability to couple the dynamic aeroservoelastic state-space model of a wing structure to a nonlinear 6-degree of freedom flight dynamic model of an aircraft to perform time simulations of the aircraft response to atmospheric gust inputs and flight control surface inputs.

Figure 23 illustrates the TTBW finite-element model in BEAM3D. The left wing and main strut are modeled as the major structural elements. The jury strut is neglected in the model as its contribution is marginal. The mass and structural properties of the finite-element model are made available to NASA by Boeing. ${ }^{19}$ The stiffness properties and the elastic definition of the wing and strut are included in the structural properties. BEAM3D models the mass and inertia properties as distributed or running mass and inertias. Thus, the point masses and inertias from the NASTRAN model provided by Boeing have to be converted into the equivalent distributed mass and inertias. Fuel weight is also modeled in the BEAM3D model. The full fuel weight corresponds to a take-off gross weight (TOGW) of 138,000 lbs. The inertias of the fuselage and tails are estimated in VSPAERO. 


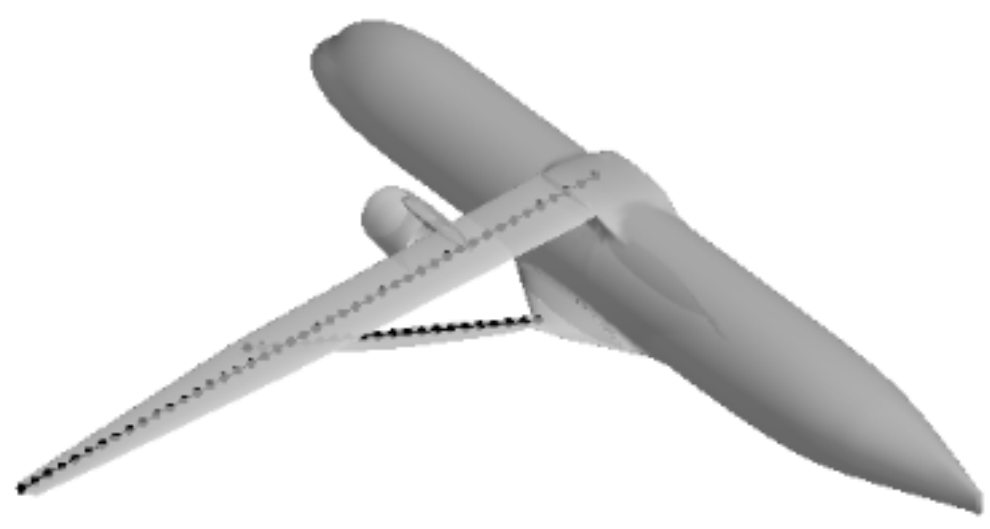

Figure 23 Finite Element Model of the Truss-Braced Wing

The NASTRAN model provided by Boeing Research \& Technology can be used to extract equivalent beam stiffnesses and running mass and inertias through individual, uncoupled beam analyses for both the wing and the strut. Cantilever boundary conditions are applied to the interior face of each beam, and four subcases are conducted for each component to isolate motion in the degree of freedom corresponding to each 3D beam stiffness. A force is applied in the spanwise direction to extract the equivalent axial stiffness, $E A$. A moment is applied about the spanwise axis in order to determine the equivalent torsional stiffness, $G J$. A force is applied in the chordwise direction to determine the equivalent chordwise bending stiffness, $E I_{z z}$. A force is applied in the flapwise direction to determine the equivalent flapwise bending stiffness, $E I_{y y}$. Deflections and loading are extracted at nodes corresponding to key geometric features to discretize beams into n-elements. The elemental deflection and loading data is then used to calculate stiffness data used by BEAM3D. There are 43 elements used for the wing and 32 elements used for the strut. The resulting stiffnesses are used to calculate deflections, and the deflection data are compared to raw NASTRAN deflection output for validation. Figure 24 shows the NASTRAN deflection of the flapwise bending stiffness loading case for the TTBW wing.

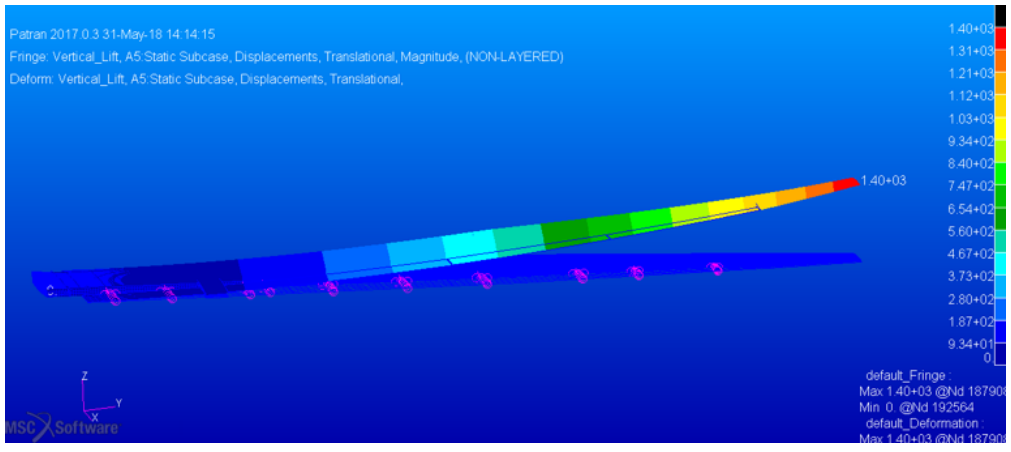

Figure 24 TTBW Wing NASTRAN Flapwise Bending Deflection

BEAM3D includes the nonlinear capability to model the tension-induced stiffening effect of the struts, which contributes to the overall stiffness matrix of the finite-element model. This is expressed as: ${ }^{21}$

$$
K_{i}(x)=K_{i}^{s}+K_{i}^{a}+K_{i}^{t}(x),
$$

where $K_{i}$ is the total element stiffness matrix of the $i$-th element, $K_{i}^{s}$ is the element structural stiffness matrix, $K_{i}^{a}$ is the element aerodynamic stiffness matrix, and $K_{i}^{t}$ is the element nonlinear stiffness matrix due to the strut tension, which is dependent on the displacement field $x(t)$ of the structure. The aerodynamic stiffness is computed from Theodorsen's theory and depends on the dynamic pressure, wing sectional lift curve slope, and other pertinent unsteady aeroelastic parameters. 


\section{B. Static Aeroelastic Analysis}

For static aeroelastic analysis, an iterative solution method is implemented for the nonlinear tension-induced stiffness, as shown in Figure 25. At each iteration between the VSPAERO model and the BEAM3D model, the iterative solution method iterates on the static deflection until it converges.

A static aeroelastic analysis is performed for the jig shape TTBW geometry. The VSPAERO model of the jig shape TTBW geometry with all the corrections is coupled to the BEAM3D model. Figure 26 shows the linear and nonlinear static aeroelastic deflections of the wings at Mach 0.745 and the design lift coefficient $C_{L}=0.73$ computed by BEAM3D. For comparison, the linear static aeroelastic deflections are also computed in the Boeing NASTRAN 3D model of the TTBW using the aerodynamic loads computed from the VSPAERO model upon convergence. Except for a minor discrepancy near the wing root, the linear static solutions of the wing bending deflection computed by NASTRAN and BEAM3D are in excellent agreement. The wing tip deflection at cruise is computed to be 23.3 inches by BEAM3D and 23.2 inches by NASTRAN. The nonlinear static solution of the wing tip deflection is computed to be 22.8 inches. Thus, the nonlinear tension-induced stiffening does not significantly alter the static aeroelastic deflections.

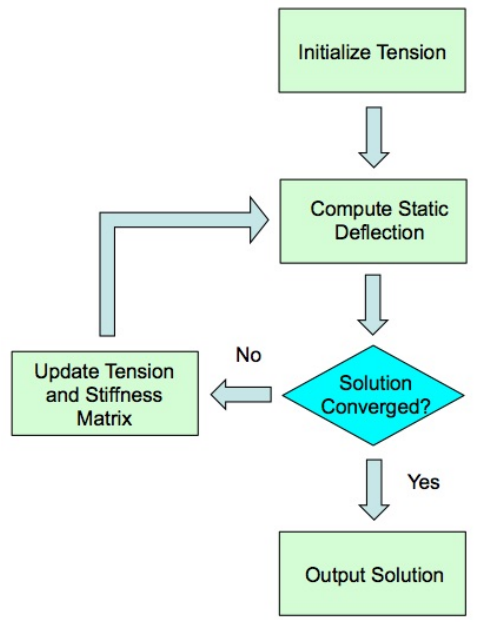

Figure 25 Iterative Solution for Nonlinear Finite-Element Model of Strut Tension Stiffening

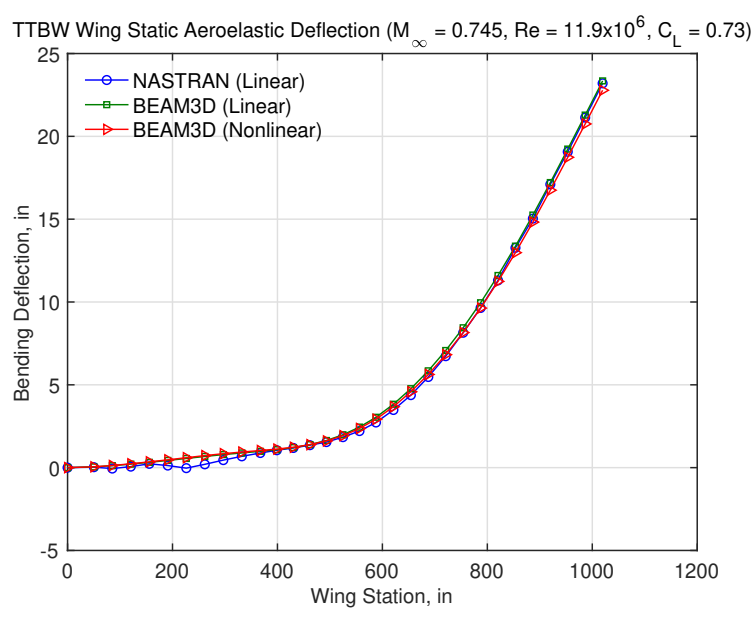

(a) Bending Deflection

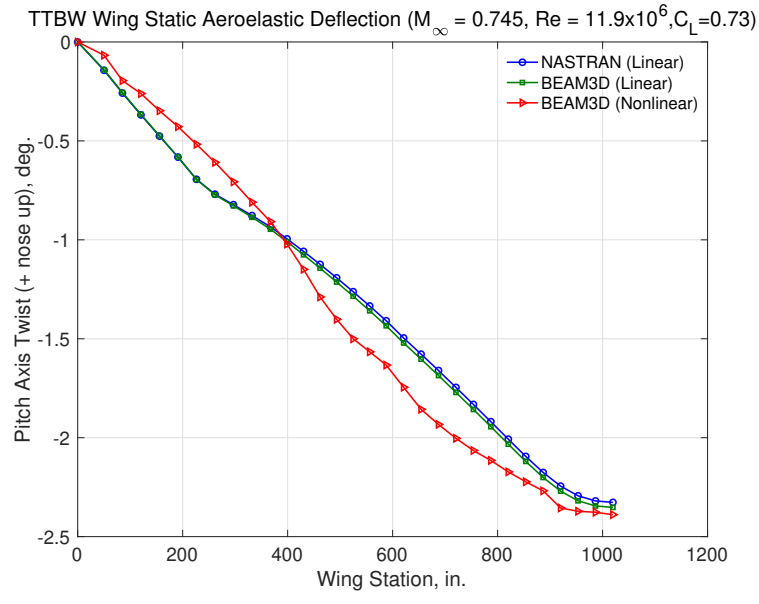

(b) Pitch Axis Twist

Figure 26 TTBW Wing Bending Deflection and Twist at Mach 0.745 and $C_{L}=0.73$ 
The nose-up wing twists about the aircraft pitch axis, or the $y$-axis, computed by BEAM3D and NASTRAN are in general agreement, but discrepancies do exist along the wing span. The wing twist computed by NASTRAN is higher than that computed by BEAM3D by at most $0.3^{\circ}$ outboard of the wing station $\mathrm{y}=400$ inches. The wing twist computed by NASTRAN is lower than that computed by BEAM3D by at most $0.2^{\circ}$ inboard of this wing station. These discrepancies could be due to the application of the distributed lift and pitching moment about the elastic axis in NASTRAN not being precisely on the elastic axis but rather along the nearest nodes. The overall nose-up wing twist at the tip is in better agreement between NASTRAN and BEAM3D with the NASTRAN computed value of $-2.39^{\circ}$ and BEAM3D computed values of $-2.35^{\circ}$ and $-2.33^{\circ}$ corresponding to the linear and nonlinear static solutions, respectively.

Figure 27 shows the strut axial displacement and tension as a function of the wing station at the design lift coefficient $C_{L}=0.73$. The axial displacement of the struts is almost 0.6 inches and the tension carried by the strut is about 171,000 lbs. $^{21}$

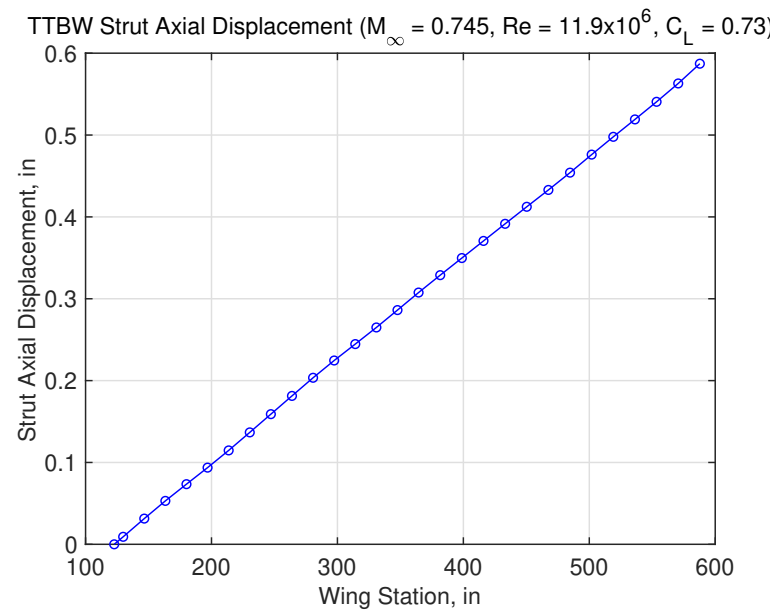

(a) Strut Axial Displacement

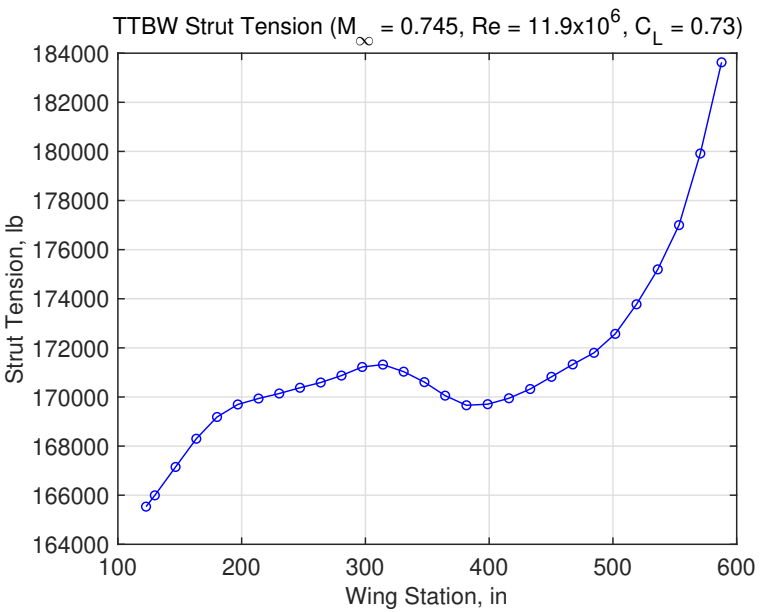

(b) Strut Tension

Figure 27 TTBW Strut Axial Displacement and Tension at Mach 0.745 and $C_{L}=0.73$

The VLM model is coupled to the transonic and viscous corrections as well as the FEA model for aero-structural iterations via the panel incidence angle using the following relationship:

$$
\tilde{\alpha}_{i n c}(y)=\alpha_{i n c}(y)+\phi_{y}(y)+\gamma(y),
$$

where $\tilde{\alpha}_{i n c}$ represents the effective panel incidence angle along the wing span, $\alpha_{i n c}$ is the jig shape incidence angle, and $\phi_{y}$ is the elastic twist about the pitch axis. The VLM and FEA models iterate until the tip twist converges. Tip twist convergence typically occurs after 2-5 iterations. If TSD/IBL coupling is not present, the virtual twist angle, $\gamma(y)$, is zero and plays no role in the aero-structural coupling; however, if both aero-structural and TSD/IBL coupling are desired, tip twist convergence is calculated for each TSD/IBL iteration at the new virtual twist angle.

A basic schematic of the full aeroelastic modeling strategy is depicted by Figure 28 . This process is used to generate the data presented in Figure 29, which shows the unlabeled plots of the lift and drag coefficients computed by the VSPAERO model of the jig shape TTBW geometry coupled to the BEAM3D model. For comparison, the lift and drag coefficients computed by the VSPAERO model of the cruise shape TTBW geometry and the wind tunnel data are also plotted. The lift and drag coefficients computed by the VSPAERO+BEAM3D model of the jig shape TTBW geometry agree very well with the VSPAERO model of the cruise shape TTBW geometry and the wind tunnel data. The drag coefficient computed by the VSPAERO+BEAM3D model actually agrees slightly better than that computed by the VSPAERO model. The lift curve of the VPSAERO model matches the slope from the wind tunnel data very well, but has a slight offset. 


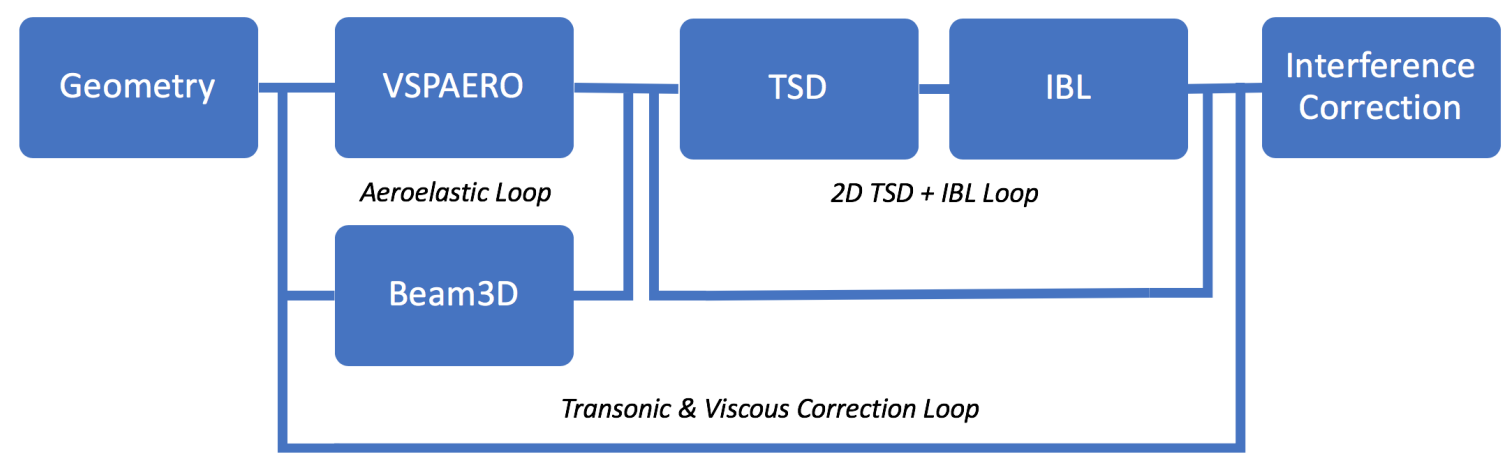

Figure 28 Full Aeroelastic Modeling Scheme

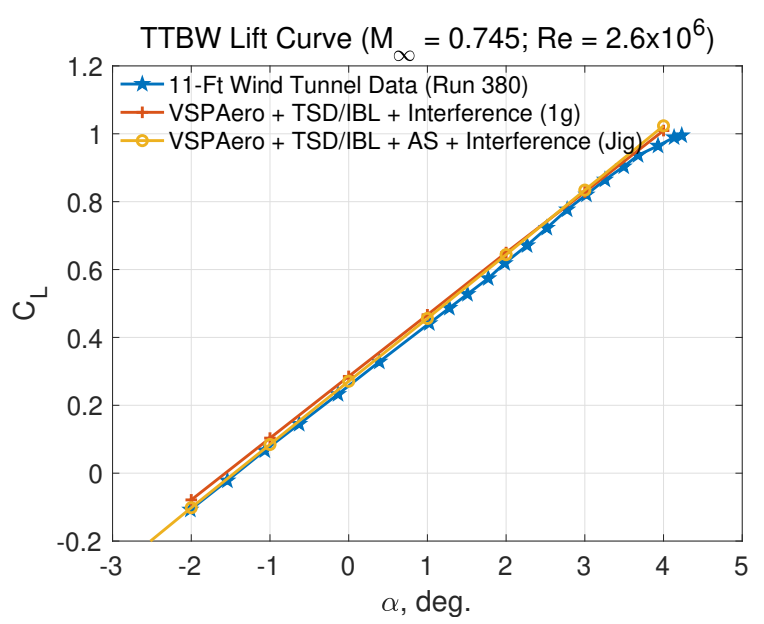

(a) Lift Curve

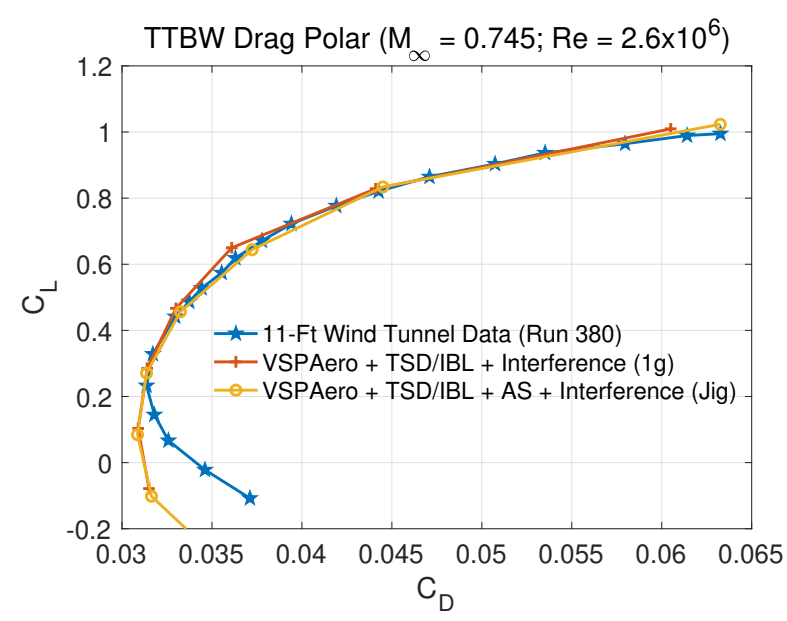

(b) Drag Polar

Figure 29 TTBW Lift Curve $C_{L}$ vs. $\alpha$ and Drag Polar $C_{L}$ vs. $C_{D}$ at Mach 0.745

\section{Frequency Analysis}

The first 12 structural dynamic modes for the TTBW computed by BEAM3D are presented for the $100 \%$ fuel condition corresponding to a TOGW of 138,000 lbs. The natural frequencies are computed with and without the tension-induced stiffening effect of the struts, referred to as $1 \mathrm{~g}$-loaded frequencies and no-load frequencies, respectively, as shown in Table 1. The BEAM3D code has been validated against NASTRAN frequency analyses. ${ }^{20}$ The lowest no-load natural frequency is $1.977 \mathrm{~Hz}$ corresponding to the first symmetric wing bending mode. For comparison, the lowest natural frequency computed by NASTRAN is $1.896 \mathrm{~Hz}$ for the same mode. The discrepancy could be due to the inconsistency in the mass used in both models. This will be verified later. The tension-induced stiffening effect increases the lowest no-load natural frequency by $2.6 \%$ to $2.028 \mathrm{~Hz}$. Figure 30 shows the 12 natural mode shapes corresponding to the first 12 modes. ${ }^{21}$ 


\begin{tabular}{|c|c|c|}
\hline Mode & No-Load Frequency $(\mathrm{Hz})$ & 1g-Loaded Frequency $(\mathrm{Hz})$ \\
\hline \hline 1st Symmetric & 1.9769 & 2.0282 \\
\hline 1st Antisymmetric & 2.0670 & 2.1066 \\
\hline 2nd Symmetric & 2.4592 & 2.5331 \\
\hline 2nd Antisymmetric & 2.4873 & 2.5468 \\
\hline 3rd Symmetric & 2.9034 & 2.9189 \\
\hline 3rd Antisymmetric & 2.8908 & 2.9242 \\
\hline 4th Symmetric & 4.4957 & 4.5194 \\
\hline 4th Antisymmetric & 5.3688 & 5.3865 \\
\hline 5th Symmetric & 6.7143 & 6.9530 \\
\hline 5th Antisymmetric & 5.5832 & 9.1957 \\
\hline 6th Symmetric & 7.0230 & 8.7344 \\
\hline 6th Antisymmetric & 9.2363 & 10.5985 \\
\hline
\end{tabular}

Table 1 TTBW Structural Dynamic Natural Frequencies Computed by BEAM3D
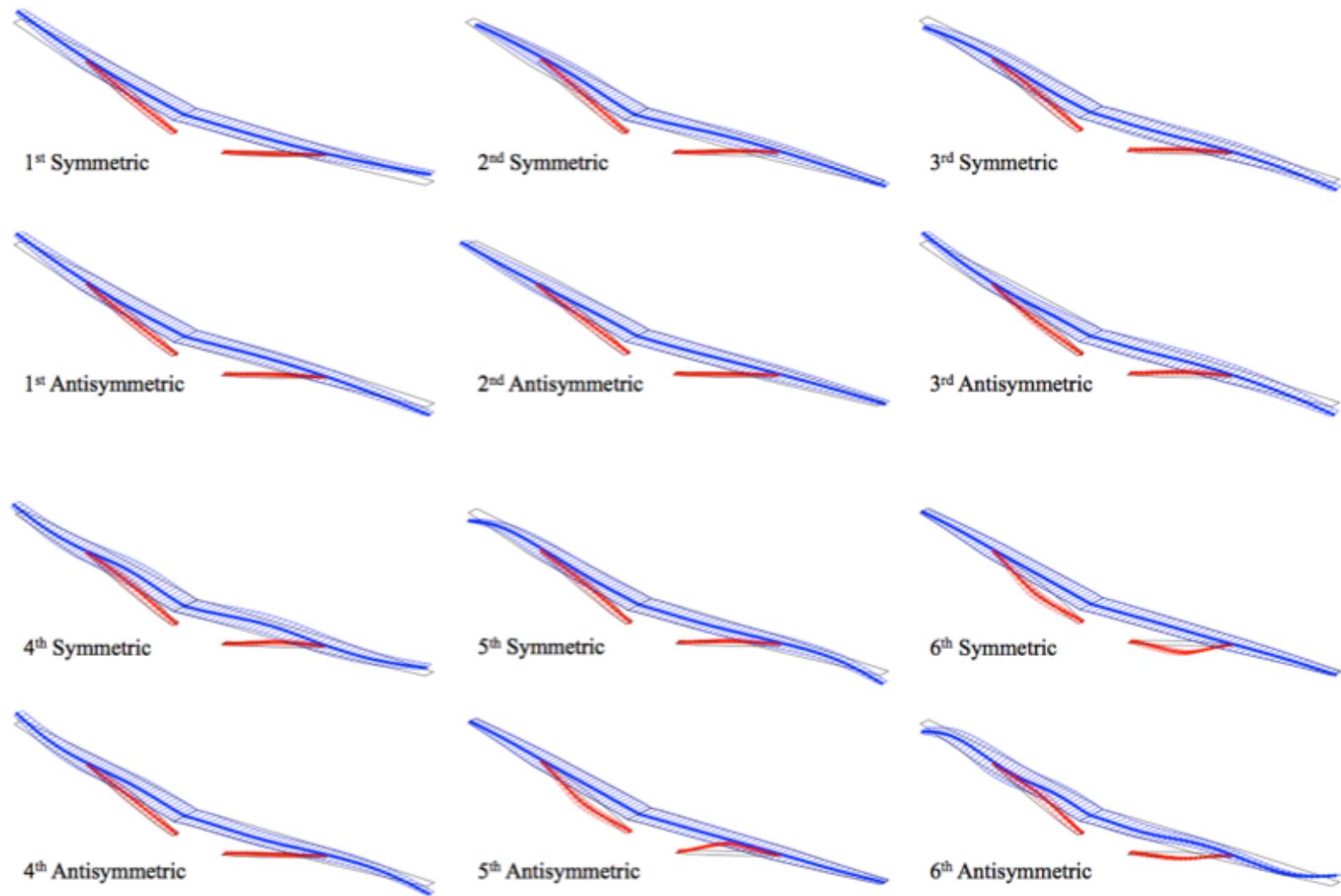

Figure 30 TTBW Natural Mode Shapes 


\section{Flight Jig Twist Optimization Study}

The jig shape OML geometry is not a flight jig shape, which accounts for the wing aeroelastic wash-out twist for the full-scale vehicle at the design flight condition, but is a wind tunnel model jig shape. Thus, the wind tunnel jig shape model is suitable for comparisons to wind tunnel data, but not necessarily suitable for generating a full flight dynamics model. An optimization strategy is developed in order to formulate a flight-optimized jig twist model.

The goal of the optimization scheme is to minimize the drag of the the aero-structural model of the jig shape for the TTBW wing. Lift constraints are added to the optimization algorithm, such that $\left|C_{L}-C_{L_{d}}\right|<0.001$, where $C_{L_{d}}$ is the design lift coefficient, 0.73. A gradient-based optimization scheme utilizing the steepest descent method is used with the cost function as described below:

$$
J(\alpha, C)=C_{D}
$$

where $C_{D}$ is the aircraft drag coefficient from the aero-structural model of the jig shape geometry and $C=\left[C_{1}, \ldots, C_{N}\right]$ is a vector of $C_{i}$, which are coefficients of a shape function that parameterize the jig twist such that:

$$
\theta(y)=\theta_{i n i}(y)+\sum_{i=1}^{N}\left(C_{i} \cdot \phi_{i}(y)\right),
$$

where $\theta(y)$ represents the jig twist distribution along the spanwise direction, $\theta_{\text {ini }}(y)$ is the initial jig twist distribution along the spanwise direction, corresponding to the wind tunnel optimized twist model, and $\phi_{i}(y)$ is a shape function of degree $i$. Shape functions are utilized in order to limit the number of design variables for optimization as well as provide a smoother converged optimized jig twist distribution. The steepest descent algorithm for updating the design variables can be represented by the following equations:

$$
\begin{gathered}
\alpha_{k+1}=\alpha_{k}-\epsilon \cdot \frac{\partial J}{\partial \alpha} \\
C_{i_{k+1}}=C_{i_{k}}-\epsilon \cdot \frac{\partial J}{\partial C_{i}} .
\end{gathered}
$$

Here, the design variable at iteration $k$ is updated based on the partial derivative of the cost function with respect to the design variable, which is calculated using a small-perturbation, second-order central difference scheme, and $\epsilon$ is a step size weight term.

The optimization procedure begins by initializing both the TTBW geometry and the parameterization of the jig twist. A baseline aero-structural solution is established by first running VSPAERO for the wind tunnel twist optimized model coupled to BEAM3D, and then iterating until the tip twist converges. Once tip twist convergence is achieved, the value of the cost function, or aircraft drag in this case, is updated. The new cost function value is compared to the convergence criteria. If the criteria are not met, small perturbations are applied to the design variables. The design jig twist, $\theta(y)$, is then calculated, and the VSPAERO mesh is deformed based on the updated twist. As before, the VSPAERO model is then coupled to BEAM3D until a converged aero-structural solution is generated. A gradient is established based on the effect of the small perturbations such that the design variables can be updated relative to their respective cost function partial derivatives. This small perturbation iterative procedure is repeated until the cost function converges. A block diagram illustrating the full jig twist optimization framework is shown in Figure 31 


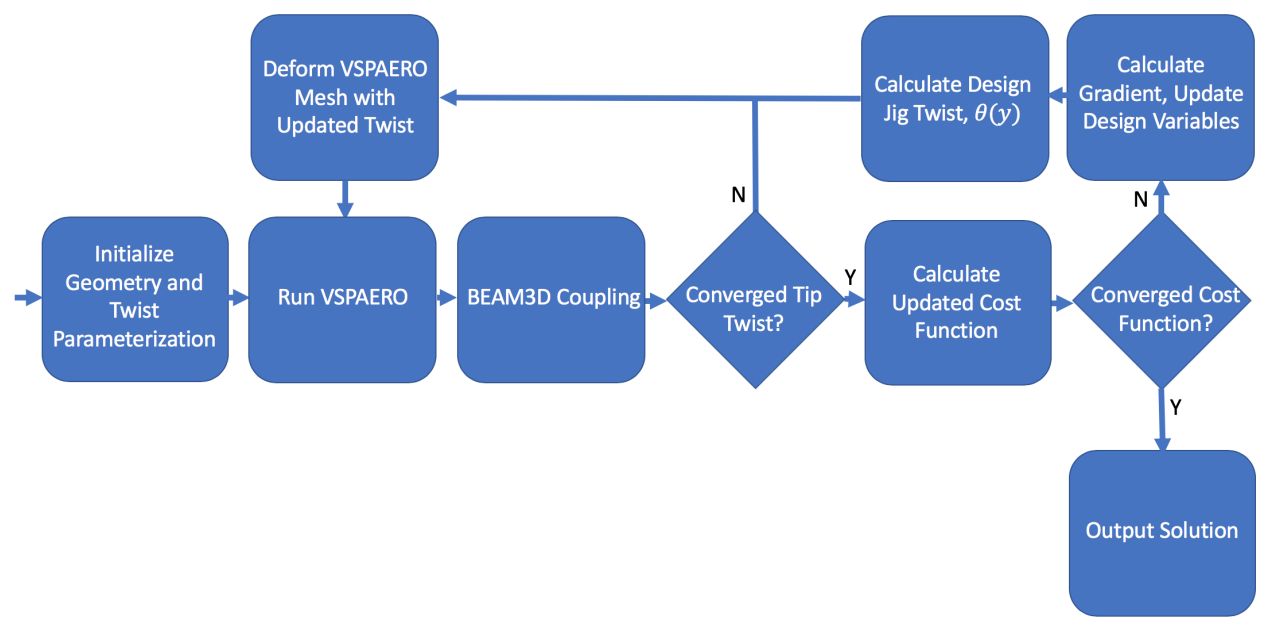

Figure 31 Jig Twist Optimization Scheme

Select results from the optimization study can be seen in Figure 32 Initially, a single, continuous shape function is utilized to parameterize twist; however, it is discovered that a piecewise polynomial, such that $\theta$ and $\frac{d \theta}{d y}$ match at the wing-strut juncture location, can provide improved jig twist optimization by accounting for aerodynamic changes at the wing-strut juncture. Of the shape function fitting techniques tested, the piecewise cubic Chebyshev polynomial fit is deemed the optimal solution. Table 2 helps to quantify the main observations for the optimal case.

\begin{tabular}{|c|c|c|c|c|}
\hline Model & $\alpha_{\text {trim }}($ deg. $)$ & $C_{L}$ & $C_{D}$ & $\sqrt{\sum_{j=1}^{n}\left(c_{L_{j}}-c_{L_{d_{j}}}\right)^{2}}$ \\
\hline \hline $1 \mathrm{~g}$ & 2.3623 & 0.7300 & 0.0393 & - \\
\hline Wind Tunnel Jig & 2.3373 & 0.7300 & 0.0397 & 0.0301 \\
\hline 3rd Order Piecewise & 2.3582 & 0.7300 & 0.0393 & 0.0203 \\
\hline
\end{tabular}

Table 2 Drag Minimization Jig Twist Optimization Results

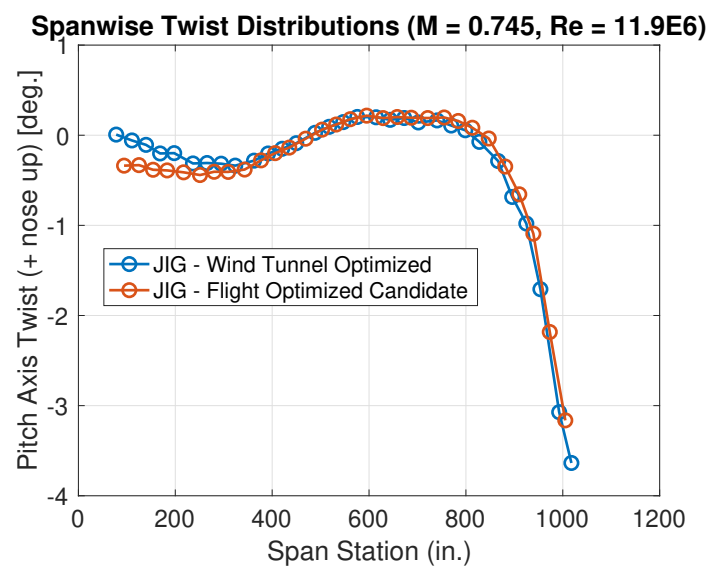

(a) Twist Distribution

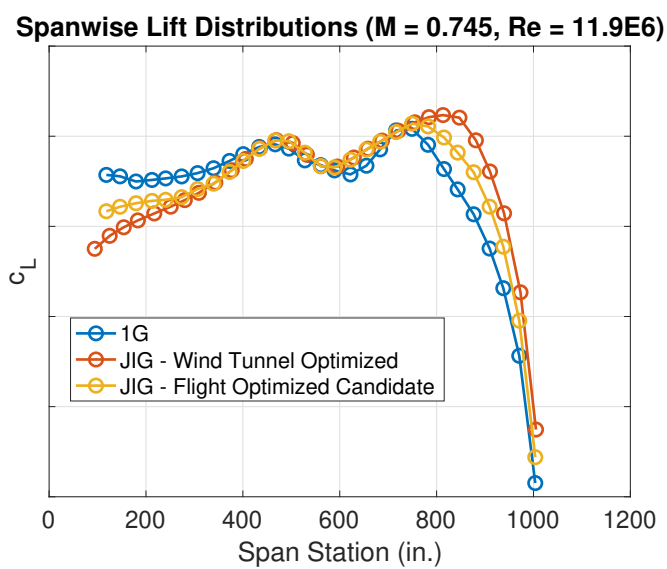

(b) Lift Distribution

Figure 32 Drag Minimization Jig Twist Optimization Candidate: 3rd Order Piecewise Chebyshev Polynomial 
Overall, the ideal optimized twist distribution maintains a significantly different $c_{L}$ distribution when compared to the 1g shape model, but it produces lower drag as expected and a reasonable optimized twist. This solution produces a reduction in lift-matching error when compared to the wind tunnel twist distribution, and it minimizes drag such that $C_{D}$ matches the value found for the $1 \mathrm{~g}$ shape model. This solution also produces a converged twist distribution that is very similar to the wind tunnel optimized twist distribution, which makes it believable. These findings suggest that the model generated could perhaps be considered a flight jig twist candidate. To ensure the flight jig twist candidate is valid, we plan to conduct a validation study using high-fidelity CFD by mapping the deformed VSPAERO mesh with flight-optimized jig twist to FUN3D in order to compare the aerodynamic performance.

\section{Conclusion}

A vortex-lattice model of the TTBW aircraft is developed. Transonic and viscous flow correction methods are implemented in the VSPAERO models to properly account for the transonic and viscous flow effects for the TTBW aircraft. A correction method for the wing-strut interference aerodynamics is developed and applied to the VSPAERO solver. A structural dynamic finite-element model, which includes the geometric nonlinear effect due to the tension in the struts causing a deflection-dependent nonlinear stiffness, of the TTBW aircraft is developed. The VSPAERO models are coupled to the finite-element model to provide a rapid capability for aero-structural modeling and flutter analysis. Both the fully corrected $1 \mathrm{~g}$ static aeroelastic model and the aero-structurally coupled, fully corrected jig shape model show excellent agreement with wind tunnel data, which validates the modeling strategies utilized. A flight-optimized jig twist model is being developed, and pending validation, it will be applied for the purpose of generating a full flight dynamic model of the TTBW aircraft.

\section{Acknowledgement}

The authors wish to acknowledge NASA Advanced Air Transport Technologies project for the funding support of this work.

\section{References}

${ }^{1}$ Bhatia, M., et. al.. "Structural and Aeroelastic Characteristics of Truss-Braced Wing: A Parametric Study," Journal of Aircraft, Vol 49, No. 1, 2012.

${ }^{2}$ Gundlach, J. F., Tetrault, P. A., Gern, F. H., Nagshineh-Pour, A. H., Ko, A., Schetz, J. A., et. al., "Conceptual Design Studies of a Strut-Braced Wing Transonic Transport,” Journal of Aircraft, Vol. 37, No. 6, 2000.

${ }^{3}$ Gur, O., Bhatia, M., Schetz, J.A., Mason, W. H., Kapania, R. K., and Mavris, D. N., "Design Optimization of a Truss-Braced Wing Transonic Transport Aircraft," Journal of Aircraft, Vol. 47, No. 6, 2010.

${ }^{4}$ Bradley, M. K. and Droney, C. K., "Subsonic Ultra Green Aircraft Research: Phase I Final Report,” NASA Contractor Report NASA/CR-2011-216847, Boeing Research and Technology, April 2011.

${ }^{5}$ Bradley, M. K., Droney, C. K., and Allen, T. J., "Subsonic Ultra Green Aircraft Research Phase II: N+4 Advanced Concept Development,” NASA Contractor Report NASA/CR-2012-217556, Boeing Research and Technology, May 2012.

${ }^{6}$ Nguyen, N., "Elastically Shaped Future Air Vehicle Concept, " NASA Innovation Fund Award 2010 Report, October 2010, Submitted to NASA Innovative Partnerships Program, http://ntrs.nasa.gov/archive/nasa/casi.ntrs.nasa.gov/20110023698.pdf

${ }^{7}$ Nguyen, N. and Urnes, J., "Aeroelastic Modeling of Elastically Shaped Aircraft Concept via Wing Shaping Control for Drag Reduction,” AIAA Atmospheric Flight Mechanics Conference, AIAA-2012-4642, August 2012.

${ }^{8}$ Urnes, Sr., J., Nguyen, N., Ippolito, C., Totah, J., Trinh, K., Ting, E., “A Mission-Adaptive Variable Camber Flap Control System to Optimize High Lift and Cruise Lift-to-Drag Ratios of Future N+3 Transport Aircraft," 51st AIAA Aerospace Sciences Meeting, AIAA-2013-0214, January 2013.

${ }^{9}$ Lebofsky, S., Ting, E., Trinh, K. V., Nguyen, N. T., "Optimization for Load Alleviation of Truss-Braced Wing Aircraft With Variable Camber Continuous Trailing Edge Flap,” 33rd AIAA Applied Aerodynamics Confererence, AIAA-2015-2723, June 2015. 
${ }^{10}$ Ting, E., Reynolds, K., Nguyen, N., and Totah, J., "Aerodynamic Analysis of the Truss-Braced Wing Aircraft Using Vortex-Lattice Superposition Approach,” 32nd AIAA Applied Aerodynamics, AIAA 2014-2597, June 2014.

${ }^{11}$ Nguyen, N., Ting, E., and Lebofsky, S., "Aeroelasticity of Axially Loaded Aerodynamic Structures for Truss-Braced Wing Aircraft," 56th AIAA/ASME/ASCE/AHS/ASC Structures, Structural Dynamics, and Materials Conference, AIAA-2015-1840, January 2015.

12 Abbott, I. H. and Von Doenhoff, A. E., Theory of Wing Sections, Dover Publications, Inc., New York, pp. 97-103.

${ }^{13}$ Fujiwara, G., Chaparro, D., and Nguyen, N., "An Integral Boundary Layer Direct Method Applied to 2D Transonic SmallDisturbance Equations,” 34th AIAA Applied Aerodynamics Conference, AIAA-2016-3160, June 2016.

${ }^{14}$ Chaparro, D., Fujiwara, G., Ting, E., and Nguyen, N., "Aerodynamic Modeling of Transonic Aircraft Using Vortex Lattice Coupled with Transonic Small Disturbance for Conceptual Design,” 34th AIAA Applied Aerodynamics Conference, AIAA-2016-3012, June 2016.

${ }^{15}$ Chaparro, D., Fujiwara, G. E., Ting, E., and Nguyen, N. T., “Transonic and Viscous Potential Flow Method Applied to Flexible Wing Transport Aircraft,” 35th AIAA Applied Aerodynamics Conference, AIAA-2017-4221, June 2017.

${ }^{16}$ Stahara, S. S., "Operational Manual for Two-Dimensional Transonic Code TSFOIL,” NASA Contractor Report 3064, December, 1978.

${ }^{17}$ Drela, M., “A User's Guide to MSES 3.05,” MIT Department of Aeronautics and Astronautics, July 2007.

18 Jordan, T. L., Langford, W. M., Belcastro, C. M., Foster, J. M., Shah, G. H., Howland, G., and Kidd, R., "Development of a Dynamically Scaled Generic Transport Model Testbest for Flight Research Experiments," AUVSI Unmanned Unlimited, Arlington, VA, 2004.

${ }^{19}$ Bradley, M. K., Droney, C. K., and Allen, T. J., "Subsonic Ultra Green Aircraft Research Phase II - Truss Braced Wing Design Exploration" Contractor Report, The Boeing Company, June 2014.

20 Ting, E., Chaparro, D., and Nguyen, N., "Development of an Integrated Nonlinear Aeroservoelastic Flight Dynamic Model of the Truss-Braced Wing Aircraft," 58th AIAA/ASME/ASCE/AHS/SC Structures, Structural Dynamics, and Materials Conference, AIAA-2017-1815, January 2017.

${ }^{21}$ Nguyen, N., Fugate, J., Xiong, J., and Kaul, U., "Flutter Analysis of the Transonic Truss-Braced Wing Aircraft Using Transonic Correction," AIAA Science and Technology Forum and Exposition, AIAA-2019-0217, January 2019. 